\title{
Performance of Quantized Random Beamforming in Delay-Tolerant Machine-Type Communication
}

\author{
Alexis A. Dowhuszko, Graciela Corral-Briones, Jyri Hämäläinen, Member, IEEE, Risto Wichman, Member, IEEE
}

\begin{abstract}
Machine-to-Machine (M2M) communication represents a new paradigm for mobile cellular networks, where a massive number of low-cost devices requests the transfer of small amounts of data without human intervention. One option to tackle this problem is obtained by combining Random Beamforming (RBF) with opportunistic scheduling. RBF can be used to induce larger channel fluctuations and opportunistic scheduling can be used to select M2M devices when their overall channel quality is good. Traditional RBF does not fulfill M2M requirements because overall channel quality needs to be tracked continuously. In order tackle this limitation, a novel codebookbased RBF architecture that identifies in advance the time instants in which overall channel quality should be reported, within a coherence time window, is proposed. This opportunistic feedback mechanism reduces signaling overhead and enables energy saving at M2M devices. A simplified methodology is presented to evaluate the system mean data rate, using for this purpose closed form formulas derived from SNR distribution approximations. Results reveal that the performance loss that is experienced for introducing the proposed modifications to traditional RBF scheme is negligible. The concepts analyzed in this paper provide useful insights, and show that codebook-based RBF with simplified opportunistic scheduling algorithms is an excellent combination to provide wide-area M2M services with low-cost devices and limited signaling overhead.
\end{abstract}

Index Terms-Machine-to-machine, range extension, random beamforming, opportunistic scheduling, limited feedback.

\section{INTRODUCTION}

The world is developing towards a networked society, where various kinds of devices will be connected forming the so-called Internet of Things. In order to support interaction and data exchange among devices without human intervention, Machine-to-Machine (M2M) communication is needed [1]. Mobile cellular networks, which already give global connectivity, are the natural option to provide network access to M2M applications. Nevertheless, since cellular networks have been originally designed for voice calls and data exchange in human-centered applications, enhancement proposals to support M2M services should take into account coexistence issues between both communication paradigms [2]. One of the main challenges in M2M communication is the massive number of devices requesting small amounts of data to be transferred. Moreover, low cost and reduced energy consumption requirements limit the number of antennas in

A. Dowhuszko is with the Centre Tecnològic de les Telecomunicacions de Catalunya (CTTC), Barcelona, Spain (e-mail: alexis.dowhuszko@cttc.es).

G. Corral-Briones is with the Institute for Advanced Studies in Engineering and Technology, Universidad Nacional de Córdoba and CONICET, Argentina (e-mail:graciela.corral@unc.edu.ar).

Jyri Hämäläinen and Risto Wichman are with Aalto University, Finland (e-mail: \{jyri.hamalainen,risto.wichman\}@aalto.fi).
M2M devices, creating serious coverage problems in locations not frequented by people (e.g., vending machines in basements, underground water/electricity monitoring systems, and traffic monitoring systems in tunnels). One potential but not widely acknowledged option to enhance quality of service in delay-tolerant M2M services is obtained by combining Random Beamforming (RBF) with opportunistic scheduling. This practical approach can reduce M2M signaling overhead and provide additional degrees of freedom to control congestion in the cellular network [3].

Multiuser Diversity (MUD) can reduce power consumption and provide coverage extension in delay-tolerant $\mathrm{M} 2 \mathrm{M}$ communication. To capitalize MUD gain in practice, the quality of the wireless channels between Base Station (BS) and M2M devices needs to be tracked and, based on this information, M2M devices should be scheduled when channel condition is good. Unfortunately, fixed device locations imply reduced fading dynamics and, as a consequence, limited MUD gain. To deal with this problem, multiple antennas can be deployed at the BS and continuous time-varying complex weights can be applied in each antenna to induce larger channel fluctuations. In the traditional RBF architecture [4], a single Downlink Pilot (DL-P) signal is used to track the overall channel quality of each user. Since this DL-P signal is affected by the RBF vector, each active user needs to feed back a new channel quality measurement every time the RBF vector changes. A similar approach was proposed in [5], where RBF vectors were selected from a common RBF codebook. Unfortunately, such RBF architecture cannot be extended for M2M services because the massive number of devices would create a huge amount of feedback.

Different methods have been proposed in the literature to reduce feedback overhead in traditional multiuser systems. An early study on the effect of SNR quantization on the performance of a single-antenna system was presented in [6], where the authors concluded that one quantization threshold is good enough when the average SNR is known a priori. Similar results were reported in [7], where it was observed that 1-bit feedback resolution is sufficient if this quantization threshold is appropriately selected according to the number of active users. This analysis was extended in [8] to Opportunistic Beamforming (OBF), where time-varying phases and powers were applied in the BS antennas. Opportunistic Beamforming requires Power Amplifiers (PAs) with linear response over the entire power variation range. However, linear PAs have poor power efficiencies and represent a serious problem in terms of cost and size. Opportunistic Cophasing (OCP) can alleviate this problem because transmit power per antenna is constant 
and only the antenna phases need to be randomly changed [9].

Selecting the M2M device with best instantaneous link quality maximizes the system data rate, but it may be unfair for those devices in bad locations. This is because M2M devices located overground (e.g., in lamp posts and traffic lights) have statistically much better channel quality than those devices with locations underground (e.g., in basements and tunnels). One option to tackle this problem is to apply Proportional Fair Scheduling (PFS), and transmit to devices when their instantaneous-to-average data rate ratio is largest. It was observed in [4] that in a RBF system with a large number of users, PFS selects with high probability a user whose channel gain vector matches the direction of the RBF vector. So, if PFS is replaced by maximum Normalized SNR (max-NSNR) scheduling, a similar system data rate will be achieved in both cases [10]. On top of that, since scheduling decisions in max-NSNR depend on the instantaneous-to-peak SNR ratio, the BS does not need to track constantly the received SNR of users. Finally, if the coherence time of the wireless channel is large and the sequence of $\mathrm{RBF}$ vectors is known, M2M devices can switch to sleep mode when detecting that their channel quality will not be good in the following time slots.

This paper presents a novel RBF architecture that can be implemented as enhancement to fulfill M2M communication requirements in contemporary cellular networks. Note that the massive number of M2M devices, along with their extended service area, reduced cost, and increased battery life, complicate the estimation of wireless channel gains in Uplink (UL). To simplify the channel tracking procedure at the M2M devices, we replace the traditional continuous-time channel randomization process [4] by a discrete-time one [11], [5]. That is, a RBF vector is selected from a common codebook that is shared between BS and M2M devices, and randomization of beamforming vectors takes place on a time-slot basis following a pseudo-random sequence that is known beforehand. M2M devices take advantage of the large coherence time of the wireless channel and estimate the individual channel gains antenna-wise, with relaxed acquisition times, using weight-free DL-P signals. On top of that, each M2M device computes the projection value between the best Transmit Beamforming (TBF) vector for the M2M device and the RBF vector of the BS. Finally, this performance indicator, which takes a finite number of discrete values, is reported to the BS to carry out maximum Projection (max-Proj) scheduling. Closed form expressions for the system data rate of different RBF schemes combined with max-Proj scheduling are derived based on approximating the received SNR with an appropriate first-order corrected version of a Chi-Squared $\left(\chi^{2}\right)$ distribution. Though the focus is put on single-beam RBF techniques [9], the proposed analysis can be extended with minor modifications to RBF scenarios with multiple simultaneous orthogonal [12], [13], [14] or non-orthogonal [15] beams.

The rest of the paper is organized as follows: Section II presents the system model and the scheduling strategies. Section III studies the performance of three different RBF schemes in presence of ideal (unquantized) feedback, and shows that OCP with max-Proj scheduling is the most attractive option. Section IV extends the OCP-Proj analysis to the

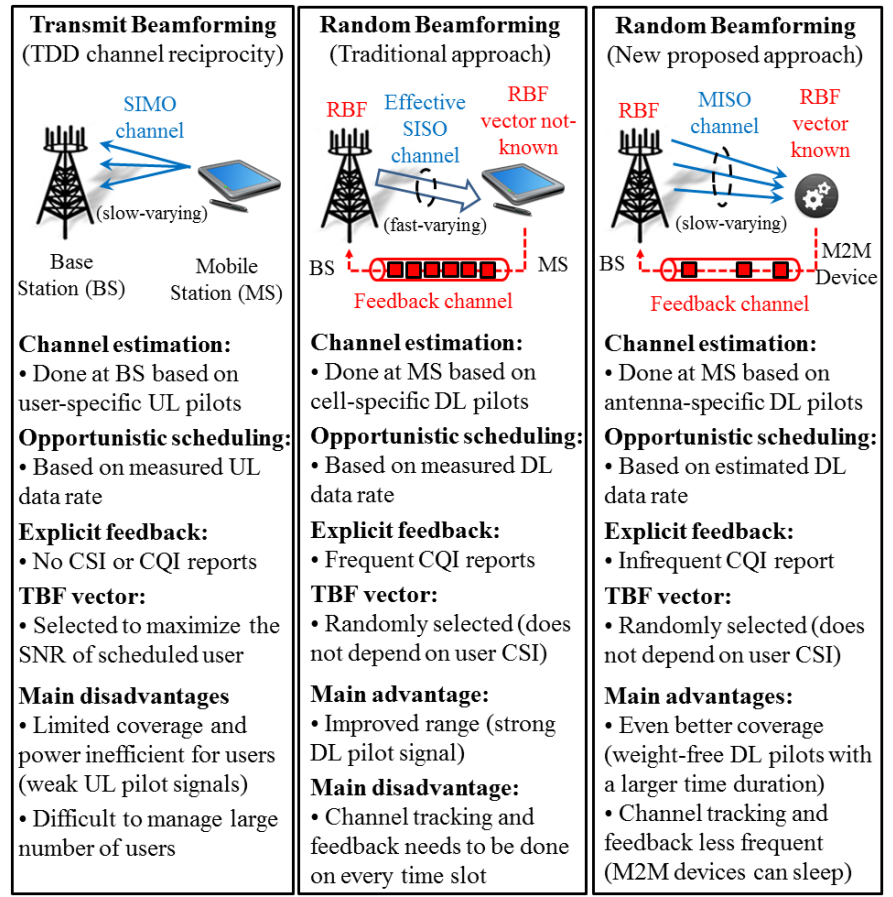

Fig. 1. Comparison of three approaches that can be used to communicate in multiuser wireless systems with TDD air interface: (a) Transmit Beamforming scheme with user-specific UL pilot signals and full Channel State Information (CSI) at BS. (b) Traditional Random Beamforming scheme with a common cell-specific DL pilot signal and full Channel Quality Information (CQI) at the BS. (c) New proposed Random Beamforming scheme with antenna-specific DL pilot signals and opportunistic CQI at the BS.

quantized feedback case, and shows that 1-bit feedback provides a negligible performance loss. Finally, conclusions are drawn in Section V. To facilitate the reading, most frequently used abbreviations are listed in Table I.

\section{SYSTEM MODEL AND ASSUMPTIONS}

Consider single-cell mobile system, where a BS equipped with $M$ antennas serves $K \gg 1$ active M2M devices with single-element antennas. From the broadband communication channel that is available for human-centric services, a narrowband channel is selected for M2M communication purposes to keep the cost of RF components low. For example, a sensible solution for an LTE system would be to select a physical resource block of bandwidth $180 \mathrm{kHz}$ around the central $1.4 \mathrm{MHz}$ portion of the band, where the Primary and Secondary Synchronization Signals (i.e., PSS and SSS) are located. To keep power consumption low at M2M devices, channel reciprocity property is set as requirement to re-use

TABLE I. List of commonly used abbreviations.

\begin{tabular}{|c|c||}
\hline max-NSNR & maximum Normalized SNR \\
\hline max-Proj & maximum Projection \\
\hline MUD & Multiuser Diversity \\
\hline OAS & Opportunistic Antenna Selection \\
\hline OBF & Opportunistic Beamforming \\
\hline OCP & Opportunistic Cophasing \\
\hline PFS & Proportional Fair Scheduling \\
\hline RBF & Random Beamforming \\
\hline TBF & Transmit Beamforming \\
\hline \hline
\end{tabular}




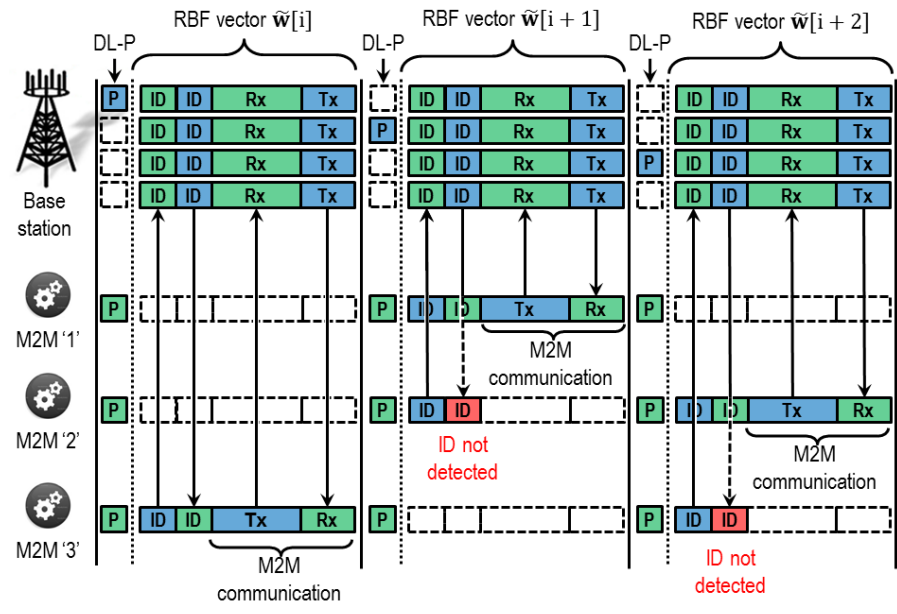

Fig. 2. Simplified M2M system with $M=4$ antennas and $K=3$ devices. Blue (green) color identifies an active transmission (reception) in the antenna element, while red color represents a denial of service. Phase 1: Channel gains are updated at M2M devices using a DL-P signal that is time-multiplexed in the BS antenna domain. Phase 2 (3): Communication requests (grants) are issued identifying M2M devices with ID numbers. Those M2M devices that do not receive a service grant in phase 3 remain silent. Phase 4 (5): Scheduled M2M device at time slot $i$ communicates in UL (DL) using RBF vector $\widetilde{\mathbf{w}}[i]$.

the same RBF vector for both DL transmit and UL receive beamforming. This way, M2M devices do not need to transmit high-power UL pilot signals to estimate the coherent combining weights per antenna at the BS. Moreover, the pilot contamination that a massive number of $\mathrm{M} 2 \mathrm{M}$ devices would create in UL is prevented. Finally, the use of DL-P signals extend the range of the cellular system by increasing the channel estimation time in those M2M devices with highest path loss. In Fig. 1 we summarize the key features of our new RBF proposal, making a comparison with previous approaches.

We focus on a half-duplex TDD air interface since channel reciprocity requirement is guaranteed if transmit and receive RF chains are calibrated. Extension to FDD is also possible if advanced channel parametrization techniques are used at the $\mathrm{BS}$, to make a reliable channel estimation in UL based on the DL feedback information that is reported by the M2M devices.

Communication between BS and M2M devices is divided into five phases as follows:

1) Channel estimation phase: Individual channel gains are estimated using DL-P signals.

2) Communication request phase: $\mathrm{M} 2 \mathrm{M}$ devices with good effective channel gains inform their ID using a common UL mini-slot (with random access contention).

3) Communication grant phase: BS makes a scheduling decision and informs the ID of the M2M device that can communicate in the remaining part of the time slot.

4) UL communication phase: The scheduled M2M device transmits information to the BS.

5) DL communication phase: The same M2M device receives information from the BS.

An illustration of this process is shown in Fig. 2. The duration of the UL and DL communication phases can be adjusted according to the traffic characteristics of the M2M services.

Communication request messages are transmitted in UL using a Random Access Channel. Therefore, those M2M

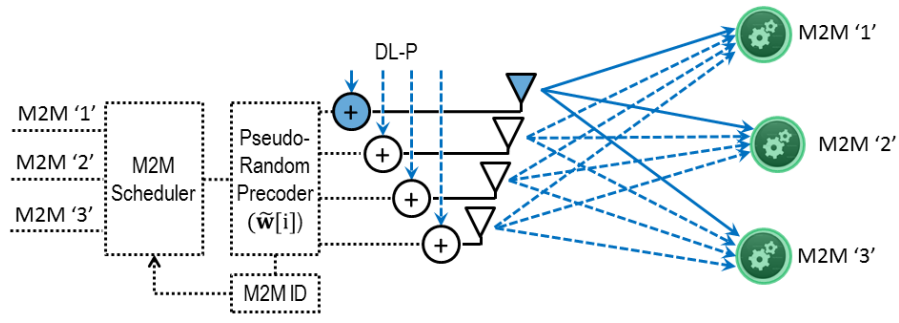

(a) Channel estimation phase (channel gains towards BS antenna '1')

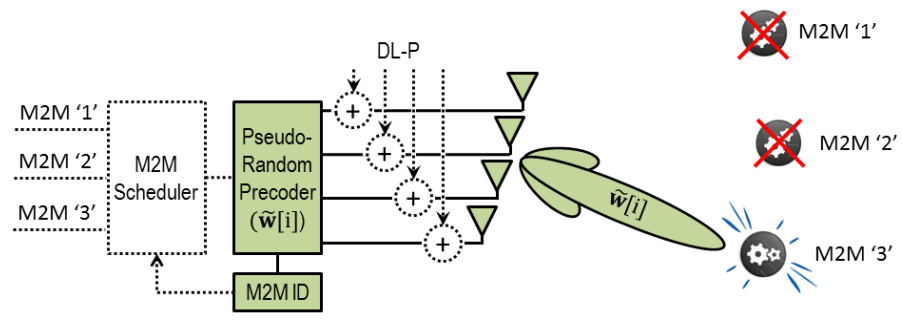

(b) UL transmission phase (M2M device ' 3 ' is active)

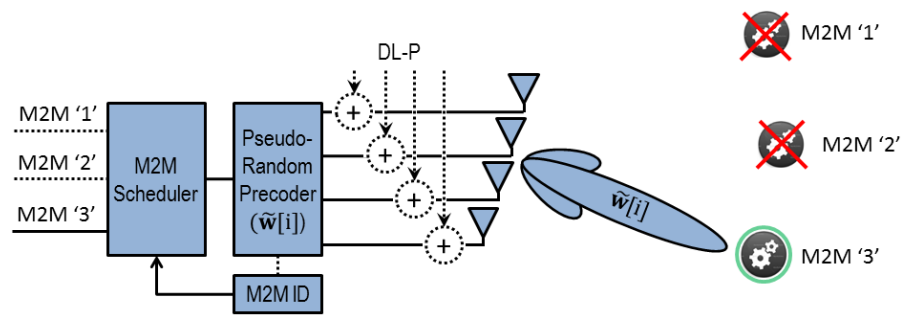

(c) DL transmission phase (M2M device '3' is active)

Fig. 3. Different phases for implementing M2M communication in a multiantenna TDD system. Individual channel gains are updated in all M2M devices in (a) channel estimation phase. Only one device is active in both (b) UL transmission phase and (c) DL transmission phases. The BS makes the scheduling decision using the information of M2M devices in the communication request phase. Blue (green) color identifies an active transmission (reception) going through the BS block or M2M device.

devices that identify that the current time slot is a good opportunity for communication, issue a random access code. Then, the BS correlates the received random access signal with each of the available codes, and grants communication to the M2M device with maximum correlation output. Therefore, if few M2M devices request communication simultaneously, it could be said that the BS selects one of them at random.

\section{A. Downlink channel estimation}

Downlink channel estimation is convenient when dealing with a massive number of M2M devices per cell. Using a common DL-P signal, channel gain vector

$$
\mathbf{h}_{k}[i]=\left(h_{k, 1}[i] \cdots h_{k, M}[i]\right) \quad k=1, \ldots, K
$$

is estimated for $\mathrm{M} 2 \mathrm{M}$ device $k$ at time $i$, where $h_{k, m}[i]$ is the normalized channel gain from BS antenna $m$. Note that only one element of channel gain vector $\mathbf{h}_{k}[i]$ is updated at each time slot, based on the antenna that transmits the DL-P signal (see Fig. 3a). Since the remaining elements of the channel gain vector are not updated, the complexity of the channel estimation block at M2M devices is the same, regardless of the number of $\mathrm{BS}$ antennas. Note that the use 
of one time-multiplexed DL-P signal per cell in the antenna domain increases the range for M2M services and, at the same time, reduces the number of orthogonal pilot signals that are needed from a multi-cell perspective. In narrowband channels with rich scattering, a flat Rayleight fading environment results. Therefore, $h_{k, m}=\left|h_{k, m}\right| e^{j \psi_{k, m}}$ can be described as a zero-mean circularly symmetric complex Gaussian Random Variable (RV) with unit variance.

\section{B. Uplink transmission with $R B F$ vector at the $B S$}

The signal that $\mathrm{BS}$ receives from $\mathrm{M} 2 \mathrm{M}$ device $k$ at time instant $i$ attains the dot product form

$$
y_{k}^{(\mathrm{ul})}[i]=\frac{\left(\mathbf{h}_{k}[i] \cdot \widetilde{\mathbf{w}}[i]\right) s_{k}^{(\mathrm{ul})}[i]}{L_{k}}+z^{(\mathrm{ul})}[i],
$$

where $\widetilde{\mathbf{w}}[i] \in \mathbb{C}^{1 \times M}$ is the RBF vector of the BS, $s_{k}^{(\mathrm{ul})}[i]$ is the complex symbol that device $k$ transmits with power $P_{k}^{(\mathrm{ul})}, L_{k}$ is the average path loss attenuation, and $z^{(\mathrm{ul})}[i]$ is zero-mean complex Additive White Gaussian Noise (AWGN) with mean power $P_{\mathrm{N}}^{(\mathrm{ul})}$. Assume that M2M devices apply open-loop UL power control to compensate the path loss attenuation. Then,

$$
P_{k}^{(\mathrm{ul})}[\mathrm{dBm}]=P_{0}^{(\mathrm{ul})}[\mathrm{dBm}]+L_{k}[\mathrm{~dB}]-G_{\mathrm{rbf}}[\mathrm{dB}],
$$

where $P_{0}^{(\mathrm{ul})}$ is the target received power in UL and $G_{\text {rbf }}$ is the mean power gain that is obtained after the RBF vector is used to combine the signals received from the different antenna branches in the analog domain (i.e., receive beamforming). To determine $G_{\mathrm{rbf}}$, the received power in a single antenna of the BS is used as reference. In practice, path loss attenuation $L_{k}$ can be estimated measuring the average received power of the DL-P signal, assuming that the corresponding transmit power is known. Note that $G_{\mathrm{rbf}}$ depends on the number of antennas $M$, the codebook design for RBF vectors, and the number of active M2M devices $K$. The larger is the value of $G_{\text {rbf }}$, the higher is the maximum path loss attenuation that the link budget of the M2M system is able to support (assuming that the maximum UL transmit power is fixed).

Due to open-loop UL power control, the BS experiences the same mean received power from all M2M devices. Therefore, from an opportunistic scheduling perspective, it is convenient to grant transmission to each $\mathrm{M} 2 \mathrm{M}$ device when the channel vector $\mathbf{h}_{k}[i]$ matches the instantaneous beamforming configuration of the RBF vector $\widetilde{\mathbf{w}}[i]$ (see Fig. 3b). Finally, it is worth noticing that since the sequence of RBF vector is known in advance, M2M devices can estimate the instantaneous receive beamforming gain $\widetilde{g}_{k}[i]=\left|\mathbf{h}_{k}[i] \cdot \widetilde{\mathbf{w}}[i]\right|^{2}$ and select an appropriate modulation and coding scheme to communicate in UL in case communication is granted during time slot $i$.

\section{Downlink transmission with RBF vector at the BS}

The signal received by scheduled $\mathrm{M} 2 \mathrm{M}$ device $k$ at time instant $i$ can be similarly written as

$$
y_{k}^{(\mathrm{dl})}[i]=\frac{\left(\mathbf{h}_{k}[i] \cdot \widetilde{\mathbf{w}}[i]\right) s_{k}^{(\mathrm{dl})}[i]}{L_{k}}+z_{k}^{(\mathrm{dl})}[i],
$$

where $s_{k}^{(\mathrm{dl})}[i]$ is the complex symbol that BS transmits in DL with power $P_{\mathrm{tx}}^{(\mathrm{dl})}$ and $z_{k}^{(\mathrm{dl})}[i]$ is zero-mean complex AWGN with mean power $P_{N}^{(\mathrm{dl})}$. Note that the same RBF vector $\widetilde{\mathbf{w}}[i]$ is used for both UL reception and DL transmission in time slot $i$ (see Fig. 3c). This is because the M2M devices do not transmit UL-P signals and, due to that, the BS does not have information on the individual channel gains per antenna. The key aspect here is that the scheduled M2M device has already estimated the relative channel phases for each antenna using DL-P signals, and has also requested to communicate in the time slot where the RBF vector $\widetilde{\mathbf{w}}[i]$ provides the appropriate phase adjustments. Since the BS does not have any information about the path loss attenuation of the scheduled device, the proper modulation and coding scheme for DL transmission should be explicitly informed by the M2M device as part of the communication request message.

\section{Scheduling strategy}

Transmitting to the M2M device with best instantaneous data rate improves the overall system performance. However, due to variations in the average path losses for the different links, this approach may not be fair for M2M devices in bad locations. To cope with this, PFS can be used [4] and communication can be granted to the device $k^{\star}$ with largest proportional rate

$$
\frac{R_{k}^{(\mathrm{dl})}[i]}{T_{k}^{(\mathrm{dl})}}=\frac{\log _{e}\left(1+\widetilde{\gamma}_{k}^{(\mathrm{dl})}[i]\right)}{\mathbb{E}\left\{\log _{e}\left(1+\widetilde{\gamma}_{k}^{(\mathrm{dl})}[i]\right)\right\}},
$$

where $R_{k}^{(\mathrm{dl})}[i]$ and $T_{k}^{(\mathrm{dl})}$ are the requested and average data rates for device $k$, respectively, while

$$
\begin{aligned}
\widetilde{\gamma}_{k}^{(\mathrm{dl})}[i] & =\bar{\gamma}_{k}^{(\mathrm{dl})} \widetilde{g}_{k}[i], \\
\bar{\gamma}_{k}^{(\mathrm{dl})} & =\frac{\mathbb{E}\left\{\left|s_{k}^{(\mathrm{dl})}[i]\right|^{2}\right\}}{L_{k} \mathbb{E}\left\{\left|z_{k}^{(\mathrm{dl})}[i]\right|^{2}\right\}}=\frac{P_{\mathrm{tx}}^{(\mathrm{dl})}}{L_{k} P_{N}^{(\mathrm{dl})}}, \\
\widetilde{g}_{k}[i] & =\left|\mathbf{h}_{k}[i] \cdot \widetilde{\mathbf{w}}[i]\right|^{2},
\end{aligned}
$$

represent the instantaneous received SNR, mean received SNR, and instantaneous SNR gain for device $k$, respectively. According to [10], PFS can be simplified by first replacing the average data rate $T_{k}^{(\mathrm{dl})}$ in (5) with the peak data rate $\log _{e}\left(1+\widehat{\gamma}_{k}^{(\mathrm{dl})}[i]\right)$, where $\widehat{\gamma}_{k}^{(\mathrm{dl})}[i]$ is the received SNR that user $k$ experiences when the best RBF codebook element is used. This step is motivated by results presented in [4], where it was shown that for a large number of users, PFS tends to schedule user $k$ when the RBF vector $\widetilde{\mathbf{w}}[i]$ is close to the beamforming configuration that corresponds to channel gain vector $\mathbf{h}_{k}[i]$. The second simplification comes by replacing the data rate with the SNR, and hence choosing the device whose instantaneous-to-peak SNR ratio is largest. Note that this approximation, whose error can be bounded studying the remainder of the first-order Taylor series expansion for the logarithmic function, is particularly good at low SNR regimes, i.e.,

$$
\frac{\log _{e}\left(1+\widetilde{\gamma}_{k}^{(\mathrm{dl})}[i]\right)}{\log _{e}\left(1+\widehat{\gamma}_{k}^{(\mathrm{dl})}[i]\right)} \approx \frac{\widetilde{\gamma}_{k}^{(\mathrm{dl})}[i]}{\widehat{\gamma}_{k}^{(\mathrm{dl})}[i]}=\frac{\widetilde{g}_{k}[i]}{\widehat{g}_{k}[i]}=m_{k}[i]
$$


The algorithm that uses (9) to perform user selection is referred to as max-NSNR. As it will be shown later, max-NSNR can be further replaced by max-Proj, where the final goal is to select the user whose projection value between the best RBF vector (of the BS codebook) and the instantaneous RBF (currently applied by the BS) is largest. Since the RBF sequence of the $\mathrm{BS}$ is known, and the best RBF vector does not change very frequently, max-Proj can implement an opportunistic feedback mechanism in a very simple way (see Fig. 1, third column).

\section{RANDOM BEAMFORMING WITH IDEAL CHANNEL STATE INFORMATION}

This section derives closed form expressions to calculate the system data rate of three RBF schemes with ideal (i.e., unquantized) feedback information at the transmitter. The key concept consists in approximating the exact Probability Density Function (PDF) for the instantaneous SNR gain $\widetilde{g}_{k^{\star}}[i]$, whose closed form expression is unknown, with a similar PDF formula that matches the first two raw moments of RV $\widetilde{g}_{k^{\star}}[i]$ and is also easy to manipulate, see [16]. More precisely, the objective is to derive tractable formulas approximating $f_{\widetilde{g}_{k^{\star}}}(x)$ with a first-order corrected version of a $\chi^{2}$ distribution with $r$ degrees of freedom, i.e.,

$$
\begin{aligned}
f_{\widetilde{g}_{k^{\star}}}(x) & \approx\left[\frac{1}{\Gamma\left(\frac{r}{2}\right)}\left(\frac{r}{2 \mathcal{G}}\right)^{\frac{r}{2}} x^{\frac{r}{2}-1} e^{-\frac{r}{2 \mathcal{G}} x}\right]\left[a_{2} x^{2}+a_{1} x+a_{0}\right], \\
r & =2\left\lfloor\mathcal{F}+\frac{1}{2}\right\rfloor,
\end{aligned}
$$

where $\Gamma(x)$ represents the Gamma function, $\lfloor x\rfloor$ is the largest integer not greater than $x$,

$$
\mathcal{G}=\mathbb{E}\left\{\widetilde{g}_{k^{\star}}[i]\right\}, \quad \mathcal{F}=\frac{\mathcal{G}^{2}}{\mathbb{E}\left\{\widetilde{g}_{k^{\star}}[i]^{2}\right\}-\mathcal{G}^{2}},
$$

constitute the so-called SNR gain and fading figure, respectively, and coefficients

$$
\begin{aligned}
& a_{2}=2\left(\frac{r}{2 \mathcal{G}}\right)^{2}\left[\left(\frac{r}{2 \mathcal{G}}\right)^{2} \frac{\mathbb{E}\left\{\widetilde{g}_{k^{\star}}[i]^{2}\right\}}{r(r+2)}-\frac{1}{4}\right], \\
& a_{1}=-(2 \mathcal{G} / r)(r+2) a_{2}, \quad a_{0}=-(\mathcal{G} / 2) a_{1}+1,
\end{aligned}
$$

are obtained according to the analysis presented in Appendix I. This approximation is attractive because the raw moments of $\mathrm{RV} \widetilde{g}_{k^{\star}}[i]$ can be obtained in closed form, as well as the subsequent derivation of the mean data rate formulas based on these moments. This is possible because the parameter $r$, which represents the degrees of freedom of the $\chi^{2}$ distribution in (10), is rounded to the closest even integer. Therefore, the definite integrals that are needed to derive the mean data rate formulas do not need to be computed numerically, but can be rather obtained by evaluating closed form expressions.

At this stage, it is possible to compute the achievable data rate of RBF scheme as follows:

$$
\begin{aligned}
& C_{k^{\star}}^{(\mathrm{ul})}(\bar{\gamma})=\mathbb{E}\left\{\log _{2}\left(1+\bar{\gamma}^{(\mathrm{ul})} \widetilde{g}_{k^{\star}}[i]\right)\right\} \\
& \quad=\log _{2}(e) \mathbb{E}\left\{\log _{e}\left(\widetilde{g}_{k^{\star}}[i]+1 / \bar{\gamma}^{(\mathrm{ul})}\right)\right\}+\log _{2}\left(\bar{\gamma}^{(\mathrm{ul})}\right)
\end{aligned}
$$

where $\bar{\gamma}_{k}^{(\mathrm{ul})}=\bar{\gamma}^{(\mathrm{ul})} \forall k$ because open loop UL power control is used. The expectation in the second line of (14) can be obtained combining (10) with equation (16) of [16], i.e.,

$$
\begin{aligned}
& \int_{0}^{\infty} \log _{e}(x+c) \beta(\beta x)^{n} e^{-\beta x} d x= \\
& =n !\left[\log _{e}(c)+e^{\beta c} \sum_{k=1}^{n+1} E_{k}(\beta c)\right] \quad \begin{array}{l}
n=0,1, \ldots \\
\beta>0 ; c>0
\end{array}
\end{aligned}
$$

where $E_{n}(x)$ represents the exponential integral function of order $n$, see (5.1.4) of [17]. So,

$$
\begin{aligned}
& \mathbb{E}\left\{\log _{e}\left(\widetilde{g}_{k^{\star}}[i]+1 / \bar{\gamma}^{(\mathrm{ul})}\right)\right\} \approx \int_{0}^{\infty} \log _{e}\left(x+1 / \bar{\gamma}^{(\mathrm{ul})}\right) \\
& \times\left[\frac{1}{\Gamma\left(\frac{r}{2}\right)}\left(\frac{r}{2 \mathcal{G}}\right)^{\frac{r}{2}} x^{\frac{r}{2}-1} e^{-\frac{r}{2 \mathcal{G}} x}\right]\left[a_{2} x^{2}+a_{1} x+a_{0}\right] d x \\
& =\left[a_{2}\left(1+\frac{2}{r}\right) \mathcal{G}^{2}+a_{1} \mathcal{G}+a_{0}\right]
\end{aligned}
$$

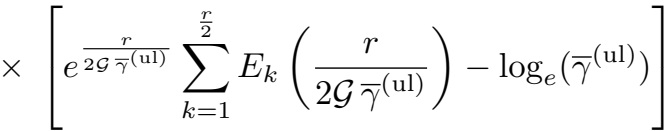

$$
\begin{aligned}
& +\left[a_{2}\left(1+\frac{2}{r}\right) \mathcal{G}^{2}+a_{1} \mathcal{G}\right] e^{\frac{r}{2 \mathcal{G} \bar{\gamma}^{(\mathrm{ul})}} E_{\frac{r}{2}}+1}\left(\frac{r}{2 \mathcal{G} \bar{\gamma}^{(\mathrm{ul})}}\right)
\end{aligned}
$$

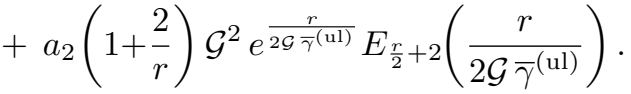

Finally, combining (14) with (16), the desired approximation is obtained. To simplify the notation, superscripts '(ul)' and '(dl)' are omitted in the remaining part of this paper.

\section{A. Opportunistic beamforming scheme}

The implementation of an OBF scheme requires the simultaneously randomizing of both amplitudes and phases of the elements of the RBF vector. In this situation, when power and phase perturbations are continuous over the entire variability range, the peak received SNR is achieved when the RBF vector is in beamforming configuration, i.e.,

$$
\widetilde{\mathbf{w}}[i]=\widehat{\mathbf{w}}_{k}[i]=\mathbf{h}_{k}[i]^{*} /\left\|\mathbf{h}_{k}[i]\right\|,
$$

where $\widehat{\mathbf{w}}_{k}[i]$ refers to the optimum beamforming vector for the actual channel state. Therefore, $\widehat{\gamma}_{k}[i]=\bar{\gamma}_{k} \widehat{g}_{k}[i]$, and

$$
\widehat{g}_{k}[i]=\left(\mathbf{h}_{k}[i] \cdot \widehat{\mathbf{w}}_{k}[i]\right)\left(\mathbf{h}_{k}[i] \cdot \widehat{\mathbf{w}}_{k}[i]\right)^{*}=\left\|\mathbf{h}_{k}[i]\right\|^{2} .
$$

Based on this analysis, it is possible to conclude that max-NSNR scheduling in the context of an OBF scheme selects at each time slot the M2M device with largest

$$
m_{k}[i]=\frac{\widetilde{\gamma}_{k}[i]}{\widehat{\gamma}_{k}[i]}=\frac{\widetilde{g}_{k}[i]}{\widehat{g}_{k}[i]}=\frac{\left|\mathbf{h}_{k}[i] \cdot \widetilde{\mathbf{w}}[i]\right|^{2}}{\left\|\mathbf{h}_{k}[i]\right\|^{2}}=\left|\widehat{\mathbf{w}}_{k}[i]^{*} \cdot \widetilde{\mathbf{w}}[i]\right|^{2} .
$$

This is equivalent to select for communication the M2M device that maximizes projection value between $\mathrm{RBF}$ vector $\widetilde{\mathbf{w}}[i]$ and optimum beamforming vector $\widehat{\mathbf{w}}_{k}[i]$. In our system model, both channel direction information $\widetilde{\mathbf{h}}_{k}[i]=\widehat{\mathbf{w}}_{k}[i]^{*}$ and channel magnitude information $\left\|\mathbf{h}_{k}[i]\right\|$ are independent. Then,

$$
\begin{aligned}
\mathbb{E}\left\{\widetilde{g}_{k^{\star}}[i]\right\} & =\mathbb{E}\left\{\left\|\mathbf{h}_{k}[i]\right\|^{2}\right\} \mathbb{E}\left\{m_{k^{\star}}[i]\right\}=M \mathbb{E}\left\{m_{k^{\star}}[i]\right\}, \\
\mathbb{E}\left\{\widetilde{g}_{k^{\star}}[i]^{2}\right\} & =\mathbb{E}\left\{\left\|\mathbf{h}_{k}[i]\right\|^{4}\right\} \mathbb{E}\left\{m_{k^{\star}}[i]^{2}\right\} \\
& =M(M+1) \mathbb{E}\left\{m_{k^{\star}}[i]^{2}\right\},
\end{aligned}
$$


since $\left\|\mathbf{h}_{k}[i]\right\|^{2}$ is $\chi^{2}$ distributed with $2 M$ degrees of freedom, while $m_{k^{*}}[i]$ is the largest order statistic of $K$ independent and identically distributed (i.i.d.) RVs with marginal Cumulative Distribution Function (CDF)

$$
F_{m_{k}}(x)=1-(1-x)^{M-1} \quad 0 \leq x \leq 1 .
$$

The latter expression is based on the fact that RV $m_{k}$ can be seen as the squared inner product between two i.i.d. isotropically unitary random vectors with $M$ elements, see [18]. Applying integration by parts in the first and second raw moments formulas, i.e.,

$$
\begin{aligned}
\mathbb{E}\left\{m_{k^{\star}}[i]\right\} & =\int_{0}^{1} x f_{m_{k^{\star}}}(x) d x \\
& =\left.\left[x F_{m_{k^{\star}}}(x)\right]\right|_{0} ^{1}-\int_{0}^{1} F_{m_{k^{\star}}}(x) d x \\
& =1-\int_{0}^{1}\left[F_{m_{k}}(x)\right]^{K} d x
\end{aligned}
$$

and

$$
\begin{aligned}
\mathbb{E}\left\{m_{k^{\star}}[i]^{2}\right\} & =\int_{0}^{1} x^{2} f_{m_{k^{\star}}}(x) d x \\
= & {\left.\left[x^{2} F_{m_{k^{\star}}}(x)\right]\right|_{0} ^{1}-\int_{0}^{1} 2 x F_{m_{k^{\star}}}(x) d x } \\
= & 1-\left.\left\{2 x \int_{0}^{x}\left[F_{m_{k}}(t)\right]^{K} d t\right\}\right|_{0} ^{1} \\
+ & 2 \int_{0}^{1} \int_{0}^{x}\left[F_{m_{k}}(t)\right]^{K} d t d x \\
= & 2 \mathbb{E}\left\{m_{k^{\star}}[i]\right\}-1+2 \int_{0}^{1} \int_{0}^{x}\left[F_{m_{k}}(t)\right]^{K} d t d x
\end{aligned}
$$

result, where

$$
\begin{array}{r}
\int_{0}^{x}\left[F_{m_{k}}(t)\right]^{K} d t=\int_{0}^{x} \sum_{k=0}^{K}\left(\begin{array}{c}
K \\
k
\end{array}\right)(-1)^{k}(1-t)^{k(M-1)} d t \\
=\sum_{k=0}^{K}\left(\begin{array}{c}
K \\
k
\end{array}\right)(-1)^{k+1} \frac{(1-x)^{k(M-1)+1}-1}{k(M-1)+1}
\end{array}
$$

and

$$
\begin{aligned}
\int_{0}^{1} \int_{0}^{x}\left[F_{m_{k}}(t)\right]^{K} d t d x=\sum_{k=0}^{K}\left(\begin{array}{l}
K \\
k
\end{array}\right)\left[\frac{(-1)^{k+1}}{k(M-1)+1}\right] \\
\times\left[\int_{0}^{1}(1-x)^{k(M-1)+1} d x-\int_{0}^{1} d x\right] \\
=\sum_{k=0}^{K}\left(\begin{array}{l}
K \\
k
\end{array}\right)\left[\frac{(-1)^{k+1}}{k(M-1)+1}\right]\left[\frac{1}{k(M-1)+2}-1\right] \\
=\sum_{k=0}^{K}\left(\begin{array}{l}
K \\
k
\end{array}\right) \frac{(-1)^{k}}{k(M-1)+2} .
\end{aligned}
$$

Combining (23)-(24) with (25)-(26) and using proposition 2.3 derived in [19], i.e.,

$$
\begin{aligned}
& \sum_{k=0}^{K}\left(\begin{array}{l}
K \\
k
\end{array}\right) \frac{(-1)^{k}}{k+\beta}=\frac{K ! \Gamma(\beta)}{\Gamma(K+1+\beta)} \\
& =\frac{K}{\beta} B(K, 1+\beta) \quad \begin{array}{l}
\beta \neq 0,1, \ldots ; \\
K=0,1, \ldots,
\end{array}
\end{aligned}
$$

where $B(x, y)$ denotes the Beta function, expressions

$$
\begin{aligned}
\mathbb{E}\left\{m_{k^{\star}}[i]\right\} & =1-K B\left(K, \frac{M}{M-1}\right), \\
\mathbb{E}\left\{m_{k^{\star}}[i]^{2}\right\} & =1+K B\left(K, \frac{M+1}{M-1}\right) \\
& -2 K B\left(K, \frac{M}{M-1}\right),
\end{aligned}
$$

are obtained. After combining (28)-(29) with (20)-(21), we get the desired closed form formulas for the first two raw moments of RV $\widetilde{\gamma}_{k^{\star}}$ in case of OBF-NSNR (same as OBF-Proj here).

1) Multiuser diversity behavior: Finally, we study the number of devices $K$ that is needed to reach a target fraction $\rho$ of the maximum SNR gain in the multiuser system. The specific value of $\rho$ can be arbitrarily selected, but in practice $\rho$ should be close to 1 . Here, we first apply $\log _{e}(x)$ function in both terms of (28). After that, we use relation $B(x, y)=\frac{\Gamma(x) \Gamma(y)}{\Gamma(x+y)}$ and a logarithmic version of Stirling's asymptotic formula, see (8.327.2) of [20]. After few manipulations, we get

$$
K(\rho) \approx\left[\frac{\Gamma\left(\frac{M}{M-1}\right)}{1-\rho}\right]^{M-1} \quad \rho \approx 1 .
$$

So, the number of devices that is needed to achieve an arbitrary (large) portion of the SNR gain available in the system grows with the power of $(M-1)$. Then, in M2M communication scenarios with a massive number of devices, a very large portion of the system MUD gain can be exploited, particularly when dealing with practical numbers of BS antennas.

\section{B. Opportunistic cophasing scheme}

Amplitude randomization leads to poor PA efficiency [4]. To cope with this, the use of OCP has been suggested in [10], where only the phases of the antennas are randomly changed. Based on this restriction, the elements of the RBF vector $\widetilde{\mathbf{w}}[i]=\left(\widetilde{w}_{1}[i], \ldots, \widetilde{w}_{M}[i]\right)$ admit expression as

$$
\widetilde{w}_{m}[i]=\frac{1}{\sqrt{M}} e^{j \widetilde{\phi}_{m}[i]} \quad m=1, \ldots, M,
$$

where random RBF phases $\left\{\widetilde{\phi}_{m}[i]: m=1, \ldots, M\right\}$ are i.i.d. uniform RVs in the interval $(-\pi, \pi]$.

Fractional SNR gain in case of OCP-NSNR has been analyzed in [10], where the authors presented a closed form expression for the conditional PDF of performance metric

$$
m_{k}[i]=\frac{\widetilde{\gamma}_{k}[i]}{\widehat{\gamma}_{k}[i]}=\frac{\widetilde{g}_{k}[i]}{\widehat{g}_{k}[i]}=\frac{\left|\sum_{m=1}^{M}\right| h_{k, m}[i]\left|e^{j\left(\psi_{k, m}[i]+\widetilde{\phi}_{m}[i]\right)}\right|^{2}}{\left(\sum_{m=1}^{M}\left|h_{k, m}[i]\right|\right)^{2}}
$$

for $M=2$, and proposed to use an exponential approximation for conditional density $f_{m_{k} \mid \mathbf{h}_{k}}(m \mid \mathbf{h})$ when the number of elements at the BS antenna array grows large (i.e., when $M \gg 2$ ). The main goal of this section is to derive tractable closed form formulas to estimate the system performance; 
therefore, we focus our attention on studying the achievable data rate of an OCP scheme that uses projection

$$
\kappa_{k}[i]=\left|\widehat{\mathbf{w}}_{k}[i]^{*} \cdot \widetilde{\mathbf{w}}[i]\right|^{2}=\frac{1}{M^{2}}\left|\sum_{m=1}^{M} e^{j\left(\psi_{k, m}[i]+\widetilde{\phi}_{m}[i]\right)}\right|^{2}
$$

to carry out scheduling decisions at the BS, instead of the normalized SNR report presented in (32). This simplification is based on the fact that, as it will be shown later, the use of $\kappa_{k}[i]$ instead of $m_{k}[i]$ provides not only similar achievable data rate performance, but it is also simpler to implement in practical wireless communication systems. Note that $m_{k}[i]$ and $\kappa_{k}[i]$ are equivalent performance indicators for the OBF case, as it was show in (19). In the rest of this section, we simplify the notation by neglecting the time index $i$.

Let $\Delta_{k, m}=\psi_{k, m}+\widetilde{\phi}_{m}$. Then, it is possible to expand

$$
\begin{aligned}
\widetilde{g}_{k} & =\left\|\mathbf{h}_{k} \cdot \widetilde{\mathbf{w}}\right\|^{2} \\
& =\left(\sum_{m=1}^{M} \frac{\left|h_{k, m}\right|}{\sqrt{M}} e^{j \Delta_{k, m}}\right)\left(\sum_{m=1}^{M} \frac{\left|h_{k, m}\right|}{\sqrt{M}} e^{j \Delta_{k, m}}\right)^{*} \\
& =\frac{1}{M} \sum_{m=1}^{M}\left|h_{k, m}\right|^{2} \\
& +\frac{2}{M} \sum_{m_{1}=1}^{M-1} \sum_{m_{2}>m_{1}}\left|h_{k, m_{1}}\right|\left|h_{k, m_{2}}\right| \operatorname{Re}\left\{e^{j\left(\Delta_{k, m_{1}}-\Delta_{k, m_{2}}\right)}\right\} .
\end{aligned}
$$

Applying the expectation operator in the previous expression, it is possible to observe that

$$
\begin{aligned}
\mathbb{E}\left\{\widetilde{g}_{k}\right\} & =\frac{1}{M} \sum_{m=1}^{M} \mathbb{E}\left\{\left|h_{k, m}\right|^{2}\right\}+\frac{2}{M} \sum_{m_{1}=1}^{M-1} \sum_{m_{2}>m_{1}} \mathbb{E}\left\{\left|h_{k, m_{1}}\right|\right\} \\
& \times \mathbb{E}\left\{\left|h_{k, m_{2}}\right|\right\} \mathbb{E}\left\{\operatorname{Re}\left\{e^{j\left(\Delta_{k, m_{1}}-\Delta_{k, m_{2}}\right)}\right\}\right\} \\
& =\frac{1}{M} \sum_{m=1}^{M} \mathbb{E}\left\{|h|^{2}\right\}+\frac{2}{M} \mathbb{E}^{2}\{|h|\} \\
& \times \mathbb{E}\left\{\sum_{m_{1}=1}^{M-1} \sum_{m_{2}>m_{1}} \operatorname{Re}\left\{e^{j\left(\Delta_{k, m_{1}}-\Delta_{k, m_{2}}\right)}\right\}\right\} \\
& =\mathbb{E}\left\{|h|^{2}\right\}+\mathbb{E}^{2}\{|h|\}\left(M \mathbb{E}\left\{\kappa_{k}\right\}-1\right) \\
& =1+\frac{\pi}{4}\left(M \mathbb{E}\left\{\kappa_{k}\right\}-1\right),
\end{aligned}
$$

since $|h|$ is a standard Rayleigh distributed RV, whose moments satisfy $\mathbb{E}\left\{|h|^{k}\right\}=\Gamma\left(\frac{k}{2}+1\right)$. Using a similar procedure, the second raw moment of $\widetilde{g}_{k}$ attains the form

$$
\mathbb{E}\left\{\widetilde{g}_{k}^{2}\right\}=\widetilde{G}_{1}+\widetilde{G}_{2}+\widetilde{G}_{3},
$$

where

$$
\begin{aligned}
\widetilde{G}_{1} & =\frac{1}{M^{2}} \mathbb{E}\left\{\left(\sum_{m=1}^{M}\left|h_{k, m}\right|^{2}\right)^{2}\right\} \\
& =\frac{1}{M^{2}} \sum_{m=1}^{M} \mathbb{E}\left\{\left|h_{k, m}\right|^{4}\right\} \\
& +\frac{4}{M^{2}} \sum_{m_{1}=1}^{M-1} \sum_{m_{2}>m_{1}} \mathbb{E}\left\{\left|h_{k, m_{1}}\right|^{2}\right\} \mathbb{E}\left\{\left|h_{k, m_{2}}\right|^{2}\right\}
\end{aligned}
$$

$$
\begin{aligned}
& \widetilde{G}_{2}=\frac{4}{M^{2}} \mathbb{E}\left\{\left(\sum_{m_{1}=1}^{M-1} \sum_{m_{2}>m_{1}}\left|h_{k, m_{1}}\right|\left|h_{k, m_{2}}\right|\right.\right. \\
& \left.\left.\times \operatorname{Re}\left\{e^{j\left(\Delta_{k, m_{1}}-\Delta_{k, m_{2}}\right)}\right\}\right)^{2}\right\} \\
& =\frac{4}{M^{2}} \sum_{m_{1}=1}^{M-1} \sum_{m_{2}>m_{1}} \mathbb{E}\left\{\left|h_{k, m_{1}}\right|^{2}\right\} \mathbb{E}\left\{\left|h_{k, m_{2}}\right|^{2}\right\} \\
& \times \mathbb{E}\left\{\operatorname{Re}^{2}\left\{e^{j\left(\Delta_{k, m_{1}}-\Delta_{k, m_{2}}\right)}\right\}\right\} \\
& +\frac{8}{M^{2}} \sum_{m_{1}=1}^{M-1} \sum_{m_{2}>m_{1}} \sum_{\substack{m_{3} \neq m_{1} \\
m_{3} \neq m_{2}}} \mathbb{E}\left\{\left|h_{k, m_{1}}\right|\right\} \\
& \times \mathbb{E}\left\{\left|h_{k, m_{2}}\right|\right\} \mathbb{E}\left\{\left|h_{k, m_{3}}\right|^{2}\right\} \\
& \times \mathbb{E}\left\{\operatorname{Re}\left\{e^{j\left(\Delta_{k, m_{3}}-\Delta_{k, m_{1}}\right)}\right\} \operatorname{Re}\left\{e^{j\left(\Delta_{k, m_{3}}-\Delta_{k, m_{2}}\right)}\right\}\right\} \\
& +\frac{8}{M^{2}} \sum_{m_{1}=1}^{M-3} \sum_{m_{2}>m_{1}} \sum_{\substack{m_{3}>m_{1} \\
m_{3} \neq m_{2}}} \sum_{\substack{m_{4}>m_{3} \\
m_{4} \neq m_{2}}} \mathbb{E}\left\{\left|h_{k, m_{1}}\right|\right\} \\
& \times \mathbb{E}\left\{\left|h_{k, m_{2}}\right|\right\} \mathbb{E}\left\{\left|h_{k, m_{3}}\right|\right\} \mathbb{E}\left\{\left|h_{k, m_{4}}\right|\right\} \\
& \times \mathbb{E}\left\{\operatorname{Re}\left\{e^{j\left(\Delta_{k, m_{1}}-\Delta_{k, m_{2}}\right)}\right\} \operatorname{Re}\left\{e^{j\left(\Delta_{k, m_{3}}-\Delta_{k, m_{4}}\right)}\right\}\right\},
\end{aligned}
$$

and

$$
\begin{aligned}
& \widetilde{G}_{3}=\frac{4}{M^{2}} \mathbb{E}\left\{\left(\sum_{m=1}^{M}\left|h_{k, m}\right|^{2}\right)\right. \\
&\left.\times\left(\sum_{m_{1}=1}^{M-1} \sum_{m_{2}>m_{1}}\left|h_{k, m_{1}}\right|\left|h_{k, m_{2}}\right| \operatorname{Re}\left\{e^{j\left(\Delta_{k, m_{1}}-\Delta_{k, m_{2}}\right)}\right\}\right)\right\} \\
&= \frac{4}{M^{2}} \sum_{m_{1}=1}^{M-1} \sum_{m_{2}>m_{1}}\left[\mathbb{E}\left\{\left|h_{k, m_{1}}\right|^{3}\right\} \mathbb{E}\left\{\left|h_{k, m_{2}}\right|\right\}\right. \\
& \quad \times \mathbb{E}\left\{\left|h_{k, m_{1}}\right|\right\} \mathbb{E}\left\{\left|h_{k, m_{2}}\right|^{3}\right\}+\mathbb{E}\left\{\left|h_{k, m_{1}}\right|\right\} \mathbb{E}\left\{\left|h_{k, m_{2}}\right|\right\} \\
&\left.\quad \times \sum_{\substack{m_{3} \neq m_{1} \\
m_{3} \neq m_{2}}} \mathbb{E}\left\{\left|h_{k, m_{3}}\right|^{2}\right\}\right] \mathbb{E}\left\{\operatorname{Re}\left\{e^{j\left(\Delta_{k, m_{1}}-\Delta_{k, m_{2}}\right)}\right\}\right\} .
\end{aligned}
$$

Then, combining (37)-(39) with (36),

$$
\begin{aligned}
\mathbb{E}\left\{\widetilde{g}_{k}^{2}\right\} & \approx \frac{\mathbb{E}\left\{|h|^{4}\right\}}{M}+\frac{(M-1)}{M} \mathbb{E}^{2}\left\{|h|^{2}\right\} \\
& +\frac{4}{M}\left(M \mathbb{E}\left\{\kappa_{k}\right\}-1\right) \\
& \times\left[\mathbb{E}\left\{|h|^{3}\right\} \mathbb{E}\{|h|\}+\frac{(M-2)}{2} \mathbb{E}^{2}\{|h|\} \mathbb{E}\left\{|h|^{2}\right\}\right] \\
& +C_{1}\left[M^{2} \mathbb{E}\left\{\kappa_{k}^{2}\right\}-2 M \mathbb{E}\left\{\kappa_{k}\right\}+1\right] \\
& =\frac{2}{M}+\frac{(M-1)}{M}+\frac{4}{M}\left(M \mathbb{E}\left\{\kappa_{k}\right\}-1\right) \\
& \times\left[\Gamma\left(\frac{5}{2}\right) \Gamma\left(\frac{3}{2}\right)+\frac{(M-2)}{2} \Gamma^{2}\left(\frac{3}{2}\right)\right] \\
& +C_{1}\left[M^{2} \mathbb{E}\left\{\kappa_{k}^{2}\right\}-2 M \mathbb{E}\left\{\kappa_{k}\right\}+1\right]
\end{aligned}
$$

results, where approximation in (40) is because coefficient

$$
\begin{aligned}
C_{1} & =\left[\frac{M(M-1)}{2} \mathbb{E}^{2}\left\{|h|^{2}\right\}+\frac{M(M-1)(M-2)}{2}\right. \\
& \left.\times \mathbb{E}^{2}\{|h|\} \mathbb{E}\left\{|h|^{2}\right\}+\frac{(M-1)(M-2)(M-3)}{2} \mathbb{E}^{4}\{|h|\}\right]
\end{aligned}
$$




$$
\begin{aligned}
& \times\left[(M-1)\left(M^{2}-3 M+3\right)\right]^{-1} \\
& =\left[M+M(M-2) \Gamma^{2}\left(\frac{3}{2}\right)+(M-2)(M-3) \Gamma^{4}\left(\frac{3}{2}\right)\right] \\
& \times\left[2\left(M^{2}-3 M+3\right)\right]^{-1}
\end{aligned}
$$

is used to simplify the final formula. We now need to study the marginal PDF of RV (33) for different values of $M$, and then apply order statistics theory to find out the first two raw moments of largest order statistic $\kappa_{k^{\star}}$ (obtained from $K$ i.i.d. samples). After that, replacing these formulas in (35) and (41), desired raw moments expressions will be obtained.

The stochastic behavior of performance metric (33) resembles the Random Walk (RW) problem introduced by Pearson in [21]. There, the author was interested in finding the PDF for the end-to-end distance $|z|$ in a chain of $n$ randomly chosen equal-length steps taken in an arbitrary direction on a two-dimensional plane. Even though the answer of this question was extensively analyzed in the literature, closed form expressions for $f_{|z|}(z)$ in terms of known functions have been derived only for $n=1,2$ and 3 : the case $n=1$ is trivial, meanwhile the formulas for $n=2$ and $n=3$ are collected in Table 2.1 of [22]. In addition, Rayleigh provided an asymptotic solution based on a two-dimensional version of the central limit theorem. This result is based on approximating $f_{|z|}(z)$ with a Rayleigh PDF, and it is perhaps the most valuable one since it serves for deriving a good approximation that fit results well, even for values of $n$ moderately large.

We are now ready to study the first two raw moments of projection (33) for different $M$. To do this, we focus on deriving the exact PDF when $M=2$, and utilize an enhanced version of Rayleigh's approximation for $M \geq 4$. Though an exact closed form solution is feasible when $M=3$, this analysis is omitted because the obtained expressions are very difficult to handle.

1) Opportunistic cophasing scheme with 2 transmit antennas: When $M=2$, we have that

$$
\begin{aligned}
\kappa_{k} & =\frac{1}{4}\left(e^{j \Delta_{k, 1}}+e^{j \Delta_{k, 2}}\right)\left(e^{-j \Delta_{k, 1}}+e^{-j \Delta_{k, 2}}\right) \\
& =\frac{1}{2}+\frac{1}{2} \cos \left(\Delta_{1,2}\right)
\end{aligned}
$$

where $\Delta_{1,2}=\left(\Delta_{k, 1}-\Delta_{k, 2}\right)$ is uniformly distributed in $(-\pi, \pi]$, or equivalently in $[0, \pi]$ since the cosine function is even. Then, the largest order statistic $\kappa_{k^{\star}}$ can be written as

$$
\begin{aligned}
\kappa_{k^{\star}} & =\max _{k \in\{1, \ldots, K\}} \kappa_{k} \\
& =\frac{1}{2}+\frac{1}{2} \cos \left(\min _{k \in\{1, \ldots, K\}} \Delta_{1,2}\right) \\
& =\frac{1}{2}+\frac{1}{2} \cos \left\{U_{(1)}\right\},
\end{aligned}
$$

where $U_{(1)}$ is scaled beta distributed according to

$$
\begin{aligned}
f_{U_{(1)}}(u) & =\frac{K}{\pi}\left(1-\frac{u}{\pi}\right)^{K-1} \\
& =\frac{K}{\pi} \sum_{k=0}^{K-1}\left(\begin{array}{c}
K-1 \\
k
\end{array}\right)\left(-\frac{1}{\pi}\right)^{k} u^{k} \quad 0 \leq u \leq \pi .
\end{aligned}
$$

By first replacing (44) into (45) and then using (3.761.10) of [20] in the resulting expression, we have that

$$
\begin{aligned}
\mathbb{E} & \left\{\kappa_{k^{\star}}\right\}=\int_{0}^{\pi}\left[\frac{1}{2}+\frac{1}{2} \cos (u)\right] f_{U_{(1)}}(u) d u \\
& =\frac{1}{2}+\frac{K}{2 \pi}\left[\sum_{k=0}^{K-1}\left(\begin{array}{c}
K-1 \\
k
\end{array}\right)\left(-\frac{1}{\pi}\right)^{k} \int_{0}^{\pi} u^{k} \cos (u) d u\right] \\
& =\frac{1}{2}+\frac{\Gamma(K+1)}{2}\left\{\sum_{k=0}^{K-1} \frac{(-1)^{k}}{\Gamma(K-k)}\right. \\
& \left.\times\left[\frac{(-1)^{\left\lfloor\frac{(k+1)}{2}\right\rfloor}}{\pi^{k+1}}\left(2\left\lfloor\frac{k+1}{2}\right\rfloor-k\right)+\sum_{l=0}^{\left\lfloor\frac{(k-1)}{2}\right\rfloor} \frac{(-1)^{l+1}}{\pi^{2(l+1)} \Gamma(k-2 l)}\right\rfloor\right\} .
\end{aligned}
$$

Using a similar procedure for the second order raw moment,

$$
\begin{aligned}
\mathbb{E} & \left\{\kappa_{k^{\star}}{ }^{2}\right\}=\int_{0}^{\pi}\left[\frac{1}{2}+\frac{1}{2} \cos (u)\right]^{2} f_{U_{(1)}}(u) d u \\
& =\frac{1}{4}+\frac{1}{2} \int_{0}^{\pi} \cos (u) f_{U_{(1)}}(u) d u \\
& +\frac{1}{4}\left[\frac{1}{2}+\frac{1}{2} \int_{0}^{\pi} \cos (2 u) f_{U_{(1)}}(u) d u\right] \\
& =\left(\mathbb{E}\left\{\kappa_{k^{\star}}\right\}-\frac{1}{8}\right)+\frac{\Gamma(K+1)}{8}\left\{\sum_{k=0}^{K-1} \frac{(-1)^{k}}{\Gamma(K-k)}\right. \\
& \left.\times\left[\frac{(-1)^{\left\lfloor\frac{(k+1)}{2}\right\rfloor}}{(2 \pi)^{k+1}}\left(2\left\lfloor\frac{k+1}{2}\right\rfloor-k\right)+\sum_{l=0}^{\left\lfloor\frac{(k-1)}{2}\right\rfloor} \frac{(-1)^{l}}{(2 \pi)^{2(l+1)} \Gamma(k-2 l)}\right]\right\} .
\end{aligned}
$$

Finally, combining (46) and (47) with (35) and (41), the desired closed form expressions for the first two raw moments of $\widetilde{g}_{k^{\star}}$ are obtained for the OCP-Proj scheme when $M=2$.

2) Opportunistic cophasing scheme with $M$ transmit antennas: Let us focus on Pearson's RW with unitary steps. In this situation, complex plane position after $M$ steps is $z=\sum_{m=1}^{M} e^{j \theta_{m}}$, where $\theta_{m}$ 's are uniform i.i.d. RVs in the interval $(-\pi, \pi]$. Then, we have that

$$
\begin{aligned}
|z|^{2} & =\left(\sum_{m_{1}=1}^{M} e^{j \theta_{m_{1}}}\right)\left(\sum_{m_{2}=1}^{M} e^{-j \theta_{m_{2}}}\right) \\
& =\sum_{m_{1}=1}^{M} \sum_{m_{2}=1}^{M} e^{j\left(\theta_{m_{1}}-\theta_{m_{2}}\right)}=M+\sum_{m_{1} \neq m_{2}} e^{j \Delta_{m_{1}, m_{2}}},
\end{aligned}
$$

where $\Delta_{m_{1}, m_{2}}=\theta_{m_{1}}-\theta_{m_{2}}$. Using a similar procedure,

$$
\begin{aligned}
|z|^{4} & =M^{2}+2 M\left(\sum_{m_{1} \neq m_{2}} e^{j \Delta_{m_{1}, m_{2}}}\right) \\
& +\left(\sum_{m_{1} \neq m_{2}} e^{j \Delta_{m_{1}, m_{2}}}\right)\left(\sum_{n_{1} \neq n_{2}} e^{-j \Delta_{n_{1}, n_{2}}}\right) \\
& =M^{2}+2 M\left(\sum_{m_{1} \neq m_{2}} e^{j \Delta_{m_{1}, m_{2}}}\right) \\
& +M(M-1)+\sum_{\substack{m_{1} \neq m_{2} ; n_{1} \neq n_{2} \\
\left(m_{1}, m_{2}\right) \neq\left(n_{1}, n_{2}\right)}} e^{j\left(\Delta_{m_{1}, m_{2}}-\Delta_{n_{1}, n_{2}}\right)}
\end{aligned}
$$


Probability distributions of both $\Delta_{m_{1}, m_{2}}$ and difference $\left(\Delta_{m_{1}, m_{2}}-\Delta_{n_{1}, n_{2}}\right)$ are even, because both RVs have equally likely positive and negative values. Then, from (48) and (49),

$$
\mathbb{E}\left\{|z|^{2}\right\}=M, \quad \mathbb{E}\left\{|z|^{4}\right\}=M(2 M-1),
$$

are obtained. Combining these results with (33), we have that

$$
\begin{aligned}
\mathbb{E}\left\{\kappa_{k}\right\} & =\frac{1}{M}, \quad \mathbb{E}\left\{\kappa_{k}^{2}\right\}=\frac{2 M-1}{M^{3}}, \\
\mathcal{F}_{\kappa_{k}} & =\frac{\mathbb{E}^{2}\left\{\kappa_{k}\right\}}{\mathbb{E}\left\{\kappa_{k}^{2}\right\}-\mathbb{E}^{2}\left\{\kappa_{k}\right\}}=\frac{M}{M-1}
\end{aligned}
$$

Note that $\mathcal{F}_{\kappa_{k}}$ tends to 1 as $M$ grows large. Therefore, in accordance with the asymptotic result for Pearson's RW, probability behavior of $\kappa_{k}$ approaches an exponential distribution when $M \rightarrow \infty$. Based on this, we decided to use a first-order corrected version for a $\chi^{2}$ distribution with 2 degrees of freedom (i.e., exponential distribution) to model the PDF of $\kappa_{k}$ in this situation. So, combining the raw moment expressions in (51) with the analysis presented in Appendix I,

$$
\begin{array}{rlr}
f_{\kappa_{k}}(x) \approx \beta e^{-\beta x}\left(a_{2} x^{2}+a_{1} x+a_{0}\right) & 0 \leq x \leq 1, \\
\beta=M, & a_{2}=-\frac{M}{4}, \quad a_{1}=1, & a_{0}=1-\frac{1}{2 M},
\end{array}
$$

results. Similarly, applying CDF definition via direct integration of previous approximation,

$$
\begin{aligned}
F_{\kappa_{k}}(x) & \approx \frac{1}{C_{2}}\left[\int_{0}^{x} a_{2} t^{2} \beta e^{-\beta t} d t+\int_{0}^{x} a_{1} t \beta e^{-\beta t} d t\right. \\
& \left.+\int_{0}^{x} a_{0} \beta e^{-\beta t} d t\right] \quad 0 \leq x \leq 1 \\
& =\frac{1}{C_{2}}\left[\frac{a_{2}}{\beta^{2}} \gamma(3, \beta x)+\frac{a_{1}}{\beta} \gamma(2, \beta x)+a_{0} \gamma(1, \beta x)\right]
\end{aligned}
$$

is obtained, where $\gamma(\alpha, x)=\int_{0}^{x} t^{\alpha-1} e^{-t} d t$ represents the lower incomplete gamma function according to (8.350.1) of [20], and $C_{2}=\left[\frac{a_{2}}{\beta^{2}} \gamma(3, \beta)+\frac{a_{1}}{\beta} \gamma(2, \beta)+a_{0} \gamma(1, \beta)\right]$ acts as normalization factor to ensure $F_{\kappa_{k}}(1)=1$. Then, re-writing lower incomplete gamma functions $\gamma(\alpha, x)$ with $\alpha>1$ in terms of $\gamma(1, x)$ using recursive relation (8.356.1) of [20], i.e.,

$$
\gamma(\alpha+1, x)=\alpha \gamma(\alpha, x)-x^{\alpha} e^{-x}
$$

and replacing $\beta$ and $\left\{a_{i}: i=0,1,2\right\}$ with values in (54), the following closed form approximation results:

$$
\begin{aligned}
F_{\kappa}(x) & \approx \frac{1}{C_{2}}\left\{\gamma(1, \beta x)\left[2 \frac{a_{2}}{\beta^{2}}+\frac{a_{1}}{\beta}+a_{0}\right]\right. \\
& \left.-e^{-\beta x}\left[a_{2} x^{2}+\left(2 \frac{a_{2}}{\beta}+a_{1}\right) x\right]\right\} \\
& =\frac{1+e^{-M x}\left(\frac{M}{4} x^{2}-\frac{1}{2} x-1\right)}{1+e^{-M}\left(\frac{M-6}{4}\right)} .
\end{aligned}
$$

At this stage, we are ready to obtain the raw moments for projection value $\kappa_{k^{\star}}$ of the scheduled M2M device, applying integration by parts. To do so, we first show that

$$
\begin{aligned}
\int_{0}^{x} & {\left[F_{\kappa_{k}}(t)\right]^{K} d t=\int_{0}^{x} \sum_{k=0}^{K}\left(\begin{array}{c}
K \\
k
\end{array}\right) e^{-M k t}\left[\frac{M}{4} t^{2}-\frac{1}{2} t-1\right]^{k} d t } \\
& \times\left[1+e^{-M}\left(\frac{M-6}{4}\right)\right]^{-K} \\
& =\left\{\sum_{k=0}^{K}\left(\begin{array}{c}
K \\
k
\end{array}\right) \sum_{\left(k_{1}, k_{2}, k_{3}\right)}\left(\begin{array}{c}
k \\
k_{1}, k_{2}, k_{3}
\end{array}\right)\left(\frac{M}{4}\right)^{k_{1}}\left(\frac{1}{2}\right)^{k_{2}}\right. \\
& \left.\times(-1)^{k_{2}+k_{3}} \int_{0}^{x} e^{-M k t} t^{2 k_{1}+k_{2}} d t\right\}\left[1+e^{-M}\left(\frac{M-6}{4}\right)\right]^{-K} \\
& =\left\{x+\sum_{k=1}^{K} \sum_{\left(k_{1}, k_{2}, k_{3}\right)}\left(\begin{array}{c}
K \\
k
\end{array}\right)\left(\begin{array}{c}
k \\
k_{1}, k_{2}, k_{3}
\end{array}\right)\left(\frac{M}{4}\right)^{k_{1}}\left(\frac{1}{2}\right)^{k_{2}}\right. \\
& \left.\times(-1)^{k_{2}+k_{3}} \frac{\gamma\left(2 k_{1}+k_{2}+1, M k x\right)}{(M k)^{2 k_{1}+k_{2}+1}}\right\}\left[1+e^{-M}\left(\frac{M-6}{4}\right)\right]^{-K}
\end{aligned}
$$

and

$$
\begin{aligned}
& \int_{0}^{1} \int_{0}^{x}\left[F_{\kappa_{k}}(t)\right]^{K} d t d x=\left\{\frac{1}{2}+\sum_{k=1}^{K} \sum_{\left(k_{1}, k_{2}, k_{3}\right)}\left(\begin{array}{c}
K \\
k
\end{array}\right)\left(\begin{array}{c}
k \\
k_{1}, k_{2}, k_{3}
\end{array}\right)\right. \\
& \times\left(\frac{M}{4}\right)^{k_{1}}\left(\frac{1}{2}\right)^{k_{2}}(-1)^{k_{2}+k_{3}} \int_{0}^{1} \gamma\left(2 k_{1}+k_{2}+1, M k x\right) d x \\
& \left.\times(M k)^{-\left(2 k_{1}+k_{2}+1\right)}\right\}\left[1+e^{-M}\left(\frac{M-6}{4}\right)\right]^{-K} \\
& =\left\{\frac{1}{2}+\sum_{k=1}^{K} \sum_{\left(k_{1}, k_{2}, k_{3}\right)}\left(\begin{array}{c}
K \\
k
\end{array}\right)\left(\begin{array}{c}
k \\
k_{1}, k_{2}, k_{3}
\end{array}\right)\left(\frac{M}{4}\right)^{k_{1}}\left(\frac{1}{2}\right)^{k_{2}}(-1)^{k_{2}+k_{3}}\right. \\
& \times\left[(M k) \gamma\left(2 k_{1}+k_{2}+1, M k\right)-\gamma\left(2 k_{1}+k_{2}+2, M k\right)\right] \\
& \left.\times(M k)^{-\left(2 k_{1}+k_{2}+2\right)}\right\} \times\left[1+e^{-M}\left(\frac{M-6}{4}\right)\right]^{-K},
\end{aligned}
$$

where $\left(k_{1}, k_{2}, k_{3}\right)$ represents all sequences of nonnegative $\left\{k_{i}: i=1,2,3\right\}$ such that their sum equals $k$, and $\left(\begin{array}{c}k \\ k_{1}, k_{2}, k_{3}\end{array}\right)$ is the multinomial coefficient. Note that (59) is due to

$$
\int \gamma(a, x) d x=x \gamma(a, x)-\gamma(a+1, x)
$$

Combining (58) and (59) with (23) and (24) first, and then these results with (35) and (41), the desired closed form approximation for the first two raw moments of $\widetilde{g}_{k^{\star}}$ are obtained when OCP-Proj scheme is used with $M \geq 4$.

\section{Opportunistic antenna selection scheme}

Finally, we consider the Opportunistic Antenna Selection (OAS) case, where the BS selects only one random antenna per time slot. Then, $\widetilde{\mathbf{w}}[i]=(0, \ldots, 0,1,0, \ldots, 0)^{T}$, where the position of the non-zero component varies randomly.

The performance of OAS-NSNR was studied in [10]. However, here we focus on using projection (33) as metric. In this situation, $\kappa_{k}[i]$ equals one if the non-zero component on $\widetilde{\mathbf{w}}[i]$ 
coincides with the antenna that experiences the best channel gain towards M2M device $k$; otherwise, it equals zero. So,

$$
f_{\kappa_{k}}(x)=\frac{M-1}{M} \delta(x)+\frac{1}{M} \delta(x-1) \quad 0 \leq x \leq 1 .
$$

In addition, since we are considering a Rayleigh fading scenario, it is possible to see that

$$
f_{\left|h_{m}\right|^{2}}(x)=\beta e^{-\beta x}, \quad F_{\left|h_{m}\right|^{2}}(x)=1-e^{-\beta x},
$$

where $\beta^{-1}=\mathbb{E}\left\{\left|h_{m}\right|^{2}\right\}=1$. Device subscript $k$ in channel gains has been dropped for notation convenience. Let $q=\left(\frac{M-1}{M}\right)$ denote the probability that $\widetilde{\mathbf{w}}[i]$ does not fit with the antenna with best channel strength. Then,

$$
\begin{aligned}
\mathbb{E}\left\{\log _{2}\left(1+\bar{\gamma} \widetilde{g}_{k^{\star}}[i]\right)\right\}=\log _{2}(\bar{\gamma})+\log _{2}(e) \\
\times\left[\left(1-q^{K}\right) \int_{0}^{\infty} \log _{e}(x+1 / \bar{\gamma}) f_{|h|_{(M)}^{2}}(x) d x\right. \\
\left.+\frac{q^{K}}{M-1} \sum_{r=1}^{M-1} \int_{0}^{\infty} \log _{e}(x+1 / \bar{\gamma}) f_{|h|_{(r)}^{2}}(x) d x\right]
\end{aligned}
$$

where

$$
\begin{aligned}
f_{|h|_{(r)}^{2}}(x) & =\frac{M !}{(r-1) !(M-r) !}\left\{\sum_{k=0}^{r-1}\left(\begin{array}{c}
r-1 \\
k
\end{array}\right)\right. \\
& \left.\times(-1)^{k} e^{-(M-r+k+1) x}\right\} \quad r=1, \ldots, M
\end{aligned}
$$

denotes the PDF of the $r$-th channel gain, when ordered from weakest to strongest for a sample size of $M$ BS antennas. At this stage, we have by formula (4.337.1) of [20] that

$$
\begin{aligned}
\int_{0}^{\infty} \log _{e}(x+1 / \bar{\gamma}) f_{|h|_{(r)}^{2}}(x) d x= \\
=\left(\begin{array}{c}
M \\
r
\end{array}\right) \sum_{k=1}^{r}\left(\begin{array}{l}
r \\
k
\end{array}\right)(-1)^{k+1} \frac{k}{M-r+k} \\
\quad \times\left\{-\log _{e}(\bar{\gamma})+e^{\frac{(M-r+k)}{\bar{\gamma}}} E_{1}\left[\frac{(M-r+k)}{\bar{\gamma}}\right]\right\} .
\end{aligned}
$$

Combining (65) with (63), desired achievable data rate formula for OAS-Proj is obtained.

\section{Performance evaluation and numerical results}

The performance of the three RBF techniques introduced so far is studied in this section assuming ideal feedback information. Performance evaluation is carried out in terms of achievable data rate as a function of the number of active devices $K$. The number of antennas at the BS is set to $M=2,4,8$, whereas the mean received SNR $\bar{\gamma}=0 \mathrm{~dB}$. The goal is to identify the scheme with the best balance between performance gain and implementation requirement costs.

According to Fig. 4, OBF represents the best choice in terms of achievable data rate, at the expense of incrementing PA design requirements. This is because the randomization of the elements of the beamforming vector should be done in both amplitudes and phases. Full range amplitude variation demands highly linear PAs, which are very poor in terms of efficiency. Therefore, along with high performance gains, comes a price to be paid in terms of hardware implementation
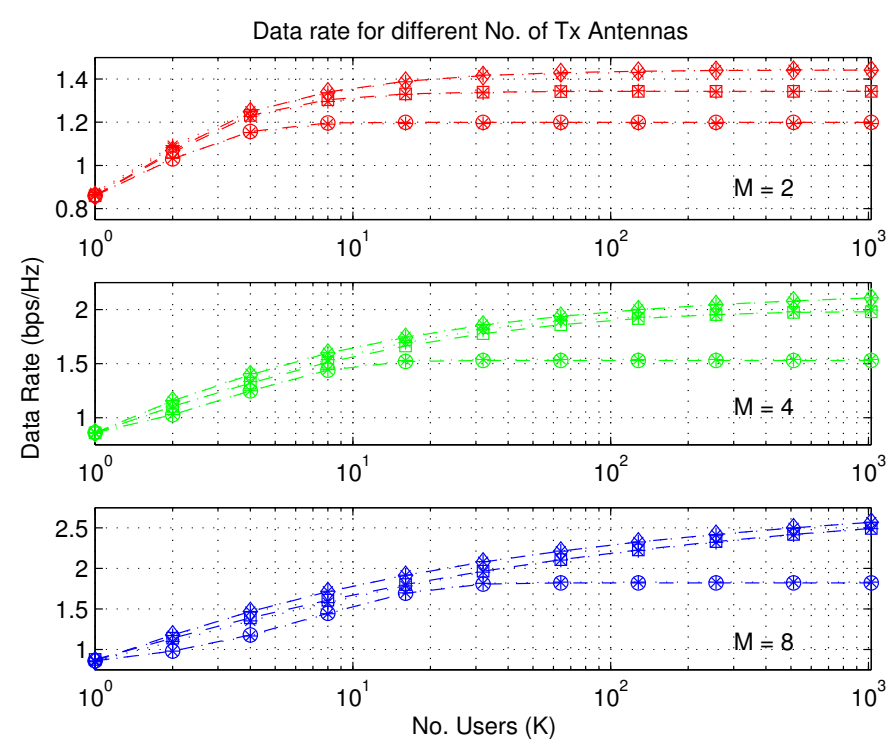

Fig. 4. Achievable data rate for different $\mathrm{RBF}$ schemes in the presence of Rayleigh fading (i.e., $\bar{\gamma}=0 \mathrm{~dB}$ ) and various numbers of BS antennas (i.e., $M=2,4,8)$. Dashed lines with circles ('o'): OAS. Dashed lines with squares (' $\square$ '): OCP. Dashed lines with diamonds (' $\diamond$ '): OBF. In all cases, point values ('*') were simulated to verify theoretical analysis.

cost. On the other hand, OAS represents the most interesting alternative with respect to hardware requirements (i.e., only one high-efficiency PA with a high-speed RF switch is needed), but it provides the poorest performance in terms of achievable data rate. In addition, OAS reaches achievable data rate saturation quickly when the number of active M2M devices increases. Therefore, the use of OAS is only advisable in a communication systems with a small number of devices, which is not typically the case of an M2M scenario.

Finally, in the middle of both extreme situations lies OCP, allowing the deployment of high-efficiency PAs without notable degradation on system performance. Note that the performance gap between $\mathrm{OBF}$ and $\mathrm{OCP}$ reduces as the number of BS antennas grows. Therefore, it is possible to conclude that OCP provides the best trade-off between achievable data rate gain and implementation costs, when analyzing the implementation of RBF in actual M2M communication scenarios.

\section{OPPORTUNISTIC COPHASING WITH QUANTIZED CHANNEL STATE INFORMATION}

In a conventional OCP scheme, the phase of each antenna varies over time continuously. Channel tracking in this situation is done via one pilot signal, broadcasted from the BS with a RBF vector that is changed on a time slot basis. Note that in practice, this random variation should be slow enough to allow an accurate estimation of the effective channel gain in reception (i.e., not very different channel states should be visited within a small time interval). This limitation imposes serious constraints on the channel dynamics that conventional OCP scheme can induce, reducing the MUD gain that can be collected in presence of slow fading channels.

Slow mobility environments are the target of RBF systems; therefore, in this work we take advantage of the large coherence time of channels to simplify system implementation 
in practice. That is, instead of tracking fast varying effective channel gains at each M2M device, each individual device will first track the slow varying individual channel gains from each antenna of the BS. After that, based on the a priori knowledge of the RBF vector that will be applied in the following scheduling interval, the M2M device will be able to estimate the effective channel gain that will be perceived [11]. Note that a similar approach has been employed in [23] to provide further MUD gain in OBF systems, by means of increasing the number of beam candidates that can be chosen when the number of devices in the system is small. At first glance, this design criteria could be interpreted as contradictory with respect to the one introduced in [4]. However, its advantages will become evident later, when channel estimation requirements and feedback quantization design will come into play in the construction of our practical RBF scheme.

\section{A. Preliminary design of quantized OCP scheme}

All active M2M devices first estimate the elements of their channel gain vectors antenna-wise, based on $M$ weight-free (omnidirectional) pilot signals that are broadcasted by the BS at the beginning of each time slot. These pilot signals are spread over $M$ consecutive scheduling intervals, and so devices update only one element of the channel gain vector per time slot. Then, each device $k$ estimates in reception the optimum beamforming vector $\widehat{\mathrm{w}}_{k}[i]$ component-wise, i.e.,

$$
\begin{aligned}
\widehat{w}_{k, m}[i] & =\frac{1}{\sqrt{M}} e^{j \widehat{\phi}_{k, m}[i]} \quad m=2, \ldots, M \\
& =\arg \max _{v_{m} \in \mathcal{V}_{p}}\left|h_{k, 1}[i]+v_{m} h_{k, m}[i]\right|,
\end{aligned}
$$

where phase quantization set

$$
\mathcal{V}_{p}=\left\{\frac{1}{\sqrt{M}} e^{j \frac{(2 n+1) \pi}{2^{N}}}: n=0, \ldots, 2^{N}-1\right\}
$$

is defined for $N$ phase quantization bits per BS antenna. Phase adjustments are done independently against the phase of the first channel gain. So, $\widehat{w}_{k, 1}[i]=1 / \sqrt{M}$ for all $i$. Based on these assumptions, it is possible to compute the peak SNR for the current channel state, i.e.,

$$
\widehat{\gamma}_{k}[i]=\bar{\gamma} \widehat{g}_{k}[i]=\bar{\gamma}\left|\mathbf{h}_{k}[i] \cdot \widehat{\mathbf{w}}_{k}[i]\right|^{2} .
$$

In the proposed quantized OCP scheme, the sum channel is varied by modifying the RBF vector $\widetilde{\mathbf{w}}[i]$ in the time domain, following a sequence that is known a priori at both extremes of the wireless link. This assumption is valid if RBF vectors belong to a finite quantization set, i.e.,

$$
\begin{aligned}
& \widetilde{\mathbf{w}}[i]=\frac{1}{\sqrt{M}}\left(1, e^{j \widetilde{\phi}_{2}[i]}, \ldots, e^{j \widetilde{\phi}_{M}[i]}\right), \\
& \frac{1}{\sqrt{M}} e^{j \widetilde{\phi}_{m}[i]} \in \mathcal{V}_{p} \quad m=2, \ldots, M,
\end{aligned}
$$

and if the pseudo-random sequence of RBF vectors is either informed to M2M devices at the beginning of the communication, or if it is stored in memory beforehand. Since each M2M device knows the pseudo-random beamforming vector that the BS will apply in the next scheduling interval, it can predict reliably the instantaneous SNR at time $i+1$, i.e.,

$$
\widetilde{\gamma}_{k}[i+1]=\bar{\gamma} \widetilde{g}_{k}[i] \approx \bar{\gamma}\left|\mathbf{h}_{k}[i] \cdot \widetilde{\mathbf{w}}[i+1]\right|^{2} .
$$

When implementing OCP-NSNR, the quantized value of

$$
\begin{aligned}
m_{k}[i] & =\frac{\widetilde{\gamma}_{k}[i+1]}{\widehat{\gamma}_{k}[i]} \\
& =\frac{|| h_{k, 1}[i]\left|+\sum_{m=2}^{M}\right| h_{k, m}[i]\left|e^{j\left(\Delta_{k, m}[i]+\varepsilon_{k, m}[i]\right)}\right|^{2}}{|| h_{k, 1}[i]\left|+\sum_{m=2}^{M}\right| h_{k, m}[i]\left|e^{j \varepsilon_{k, m}[i]}\right|^{2}}
\end{aligned}
$$

needs to be reported from the M2M device to the BS, where $\Delta_{k, m}[i]=\widetilde{\phi}_{m}[i+1]-\widehat{\phi}_{k, m}[i]$ is the phase difference between the $m$-th component of both $\widetilde{\mathbf{w}}[i+1]$ and $\widehat{\mathbf{w}}_{k}[i]$ vectors, while $\varepsilon_{k, m}[i]=\psi_{k, m}[i]+\widehat{\phi}_{k, m}[i]$ is the quantization noise that is generated for using a finite number of phase bits.

The effect of feedback quantization on the performance of OBF-NSNR scheme has been analyzed in [24]. However, since in this paper we consider that there is no amplitude ramdomization on the elements of the transmit beamforming vector $\widetilde{\mathbf{w}}[i]$, we are interested in:

1) The feedback quantization analysis for OCP-NSNR,

2) The impact that feedback quantization has on OCP-Proj when using

$$
\kappa_{k}[i]=\left|\widehat{\mathbf{w}}_{k}[i]^{*} \cdot \widetilde{\mathbf{w}}[i+1]\right|^{2}=\frac{1}{M}\left|1+\sum_{m=2}^{M} e^{j \Delta_{k, m}[i]}\right|^{2}
$$

as feedback report from each device $k$ to the BS.

Note that OCP-NSNR is equivalent to OCP-Proj if channel estimation in the receiver only considers the phase portion of channel coefficients. Finally, because we focus on environments with slow- or even no-mobility, channel estimates become reliable, and the notation is simplified again by neglecting the time index $i$ in the remaining of this section.

\section{B. Feedback characterization for OCP-Proj scheme}

This section summarizes different useful results, previously presented in [11], which characterize feedback overhead and RBF precoder probabilities when implementing OCP-Proj. Let $\mathcal{K}$ be the set of all possible values that projection (73) can take when the components of both beamforming vector $\widehat{\mathbf{w}}_{k}$ and RBF vector $\widetilde{\mathbf{w}}$ belong to the same quantization set $\mathcal{V}_{p}$.

Let us start with $N=1$ phase quantization bits. In this situation, the set of projection values was reported to be

$$
\mathcal{K}=\left\{\begin{array}{cl}
(2 l)^{2} / M^{2} & \text { for } M \text { even } \\
(2 l+1)^{2} / M^{2} & \text { for } M \text { odd }
\end{array} l=0, \ldots,\lfloor M / 2\rfloor\right\} .
$$

Note that the number of elements $|\mathcal{K}|=\lceil(M+1) / 2\rceil$ is crucial to determine the required capacity of the feedback channel. In addition, it was also observed that the probability of reporting a given feedback codeword $\mathbf{b}_{\kappa}$ in this situation is

$$
\operatorname{Pr}\left\{\mathbf{b}_{\kappa}\right\}=\frac{B_{1}(\kappa)}{2^{M}}\left(\begin{array}{c}
M \\
M(1-\sqrt{\kappa}) / 2
\end{array}\right),
$$


with

$$
B_{1}(\kappa)= \begin{cases}1 & \text { for } \kappa=0 \\ 2 & \text { otherwise. }\end{cases}
$$

Similarly, when $N=2$, it was observed that the set of possible projection values becomes

$$
\begin{gathered}
\mathcal{K}= \begin{cases}{\left[\left(2 l_{x}\right)^{2}+\left(2 l_{y}\right)^{2}\right] /\left[2 M^{2}\right]} & \text { for } M \text { even } \\
{\left[\left(2 l_{x}+1\right)^{2}+\left(2 l_{y}+1\right)^{2}\right] /\left[2 M^{2}\right]} & \text { for } M \text { odd }\end{cases} \\
\left.: l_{x}=0, \ldots,\lfloor M / 2\rfloor ; l_{y}=0, \ldots, l_{x}\right\}
\end{gathered}
$$

where its cardinality $|\mathcal{K}|=1 / 2\lceil(M+1) / 2\rceil\lceil(M+3) / 2\rceil$. Finally, it was also showed that the probability of reporting feedback codeword $\mathbf{b}_{\kappa}$ in this situation attains expression

$$
\begin{aligned}
\operatorname{Pr}\left\{\mathbf{b}_{\kappa}\right\} & =\frac{B_{2}\left(\kappa_{x}, \kappa_{y}\right)}{4^{M}}\left(\begin{array}{c}
M \\
M\left(1-\sqrt{\kappa_{x}}\right) / 2
\end{array}\right) \\
& \times\left(\begin{array}{c}
M \\
M\left(1-\sqrt{\kappa_{y}}\right) / 2
\end{array}\right) \quad \kappa=\left(\kappa_{x}+\kappa_{y}\right) / 2,
\end{aligned}
$$

with both components $\kappa_{x}$ and $\kappa_{y}$ (with $\kappa_{y} \leq \kappa_{x}$ ) belonging to the set defined in (74), and

$$
B_{2}\left(\kappa_{x}, \kappa_{y}\right)= \begin{cases}1 & \text { for } \kappa_{x}=\kappa_{y}=0 \\ 4 & \text { for } \kappa_{x}=\kappa_{y} \neq 0 \text { or } \kappa_{x}, \kappa_{y}=0, \\ 8 & \text { otherwise }\end{cases}
$$

Unfortunately, the characterization of the feedback information for other values of $N$ is not simple. To the best of our knowledge, it is not possible to obtain a general closed form expressions for the different values and probabilities that projection $\kappa_{k}$ can take when $N \geq 3$. To cope with this limitation, continuous (truncated) exponential distribution introduced in Section III-B2 is used to model the actual PDF behavior of $f_{\kappa_{k}}(x)$ when a discrete analysis is not tractable. Note that the derived closed form approximation will be asymptotically tight as the number of BS antennas $M$ grows.

Finally, it is observed that when $M=2$, the set of possible projection values becomes

$$
\mathcal{K}=\left\{\cos ^{2}\left(\frac{\pi l}{2^{N}}\right): l=0, \ldots, 2^{N-1}\right\},
$$

with $|\mathcal{K}|=2^{N-1}+1$ and

$$
\operatorname{Pr}\left\{\mathbf{b}_{\kappa}\right\}=\left\{\begin{array}{cl}
2^{-N} & \text { for } \kappa=0 \text { or } \kappa=1 \\
2^{-N+1} & \text { otherwise. }
\end{array}\right.
$$

\section{Effect of parameter $N$ on SNR gain of OCP-Proj scheme}

Let us assume that OCP-Proj is implemented, such that the elements of $\widehat{\mathbf{w}}_{k}$ and $\widetilde{\mathbf{w}}$ belong to the same quantization set $\mathcal{V}_{p}$. In this case, the SNR gain of a randomly selected device is

$$
\begin{aligned}
\mathbb{E}\left\{\widetilde{g}_{k}\right\} & =\frac{1}{M} \sum_{m=1}^{M} \mathbb{E}\left\{\left|h_{k, m}\right|^{2}\right\} \\
& +\frac{2}{M} \sum_{m_{1}=1}^{M-1} \sum_{m_{2} \neq m_{1}} \mathbb{E}\left\{\left|h_{k, m_{1}}\right|\left|h_{k, m_{2}}\right|\right\} \\
& \times \mathbb{E}\left\{\operatorname{Re}\left\{e^{j\left(\Delta_{k, m_{1}}-\Delta_{k, m_{2}}+\varepsilon_{k, m_{1}}-\varepsilon_{k, m_{2}}\right)}\right\}\right\}
\end{aligned}
$$

According to the cosine summation formula, we have that

$$
\begin{aligned}
\mathbb{E}\{\operatorname{Re} & \left.\left\{e^{j\left(\Delta_{k, m_{1}}-\Delta_{k, m_{2}}+\varepsilon_{k, m_{1}}-\varepsilon_{k, m_{2}}\right)}\right\}\right\}= \\
= & \mathbb{E}\left\{\cos \left(\Delta_{k, m_{1}}-\Delta_{k, m_{2}}\right)\right\} \mathbb{E}\left\{\cos \left(\varepsilon_{k, m_{1}}-\varepsilon_{k, m_{2}}\right)\right\} \\
- & \mathbb{E}\left\{\sin \left(\Delta_{k, m_{1}}-\Delta_{k, m_{2}}\right)\right\} \mathbb{E}\left\{\sin \left(\varepsilon_{k, m_{1}}-\varepsilon_{k, m_{2}}\right)\right\} .
\end{aligned}
$$

Phase adjustment is done using the first channel as reference; so, quantization error $\varepsilon_{k, 1}=0$, while $\left\{\varepsilon_{k, m}: m=2, \ldots, M\right\}$ are i.i.d. uniform RVs in interval $\mathcal{I}=\left(-\pi / 2^{N}, \pi / 2^{N}\right]$. So,

$$
\begin{aligned}
\mathbb{E}\left\{\cos \left(\varepsilon_{k, m}\right)\right\} & =\frac{2^{N-1}}{\pi} \int_{\mathcal{I}} \cos (\theta) d \theta \\
& =\frac{2^{N}}{\pi} \sin \left(\frac{\pi}{2^{N}}\right):=C_{N} \quad m=2, \ldots, M,
\end{aligned}
$$

$\mathbb{E}\left\{\cos \left(\varepsilon_{k, m_{1}}-\varepsilon_{k, m_{2}}\right)\right\}=C_{N}^{2}$

$$
m_{1}=2, \ldots, M-1 ; m_{2}=m_{1}+1, \ldots, M \text {. }
$$

In addition, it is also possible to observe that

$$
\begin{array}{rl}
\mathbb{E}\left\{\sin \left(\varepsilon_{k, m}\right)\right\}=0 & m=2, \ldots, M, \\
\mathbb{E}\left\{\sin \left(\varepsilon_{k, m_{1}}-\varepsilon_{k, m_{2}}\right)\right\}=0 \quad m_{1}=1, \ldots, M-1 ; \\
& m_{2}=m_{1}+1, \ldots, M,
\end{array}
$$

because the sine function is an odd function. Then, combining these expressions with (82)-(83), we arrive at

$$
\mathbb{E}\left\{\widetilde{g}_{k}\right\}=1+\frac{\pi}{4}\left(M \mathbb{E}\left\{\kappa_{k}\right\}-1\right) C_{N}\left[\frac{2}{M}+\left(\frac{M-2}{M}\right) C_{N}\right]
$$

Max-Proj scheduling is used to select device $k^{\star}$ with the largest projection metric, i.e.,

$$
\kappa_{k^{\star}}=\max _{k=\{1, \ldots, K\}} \kappa_{k}
$$

Therefore,

$$
\mathbb{E}\left\{\kappa_{k^{\star}}\right\}=1-\int_{0}^{1}\left[F_{\kappa_{k}}(x)\right]^{K} d x
$$

where

$$
\begin{aligned}
F_{\kappa_{k}}(x) & =\int_{0}^{x} f_{\kappa_{k}}(x) d x, \\
f_{\kappa_{k}}(x) & =\sum_{\kappa \in \mathcal{K}} \operatorname{Pr}\left\{\mathbf{b}_{\kappa}\right\} \delta(x-\kappa) \quad 0 \leq x \leq 1,
\end{aligned}
$$

are the $\mathrm{CDF}$ and $\mathrm{PDF}$ of $\mathrm{RV} \kappa_{k}$, respectively. Individual channel gains $\left\{\left|h_{k, m}\right|: m=1, \ldots, M\right\}$ and phase quantization errors $\left\{\varepsilon_{k, m}: m=1, \ldots, M\right\}$ do not depend on the values that $\kappa_{k}$ takes. Based on this, and combining (90) with (91)-(92), we get the desired result.

Figure 5 shows the SNR gain of OCP-Proj scheme as a function of the number of active devices $K$ for $M=2,4,8$ and $N=1,2,3$. The upper bound with ideal feedback (i.e., when $N \rightarrow \infty$ bits) is also included for sake of comparison. As expected, the SNR gain that OCP-Proj scheme provides grows with $N$, see (88). However, it is worth noticing that most of the available MUD gain is already obtained when $N=3$; in this situation, OCP-Proj reaps between $95 \%$ and $97.5 \%$ of the SNR gain of OCP-Proj with ideal phase information. In addition, the number of active devices $K$ that OCP-Proj requires to achieve an arbitrary large portion 

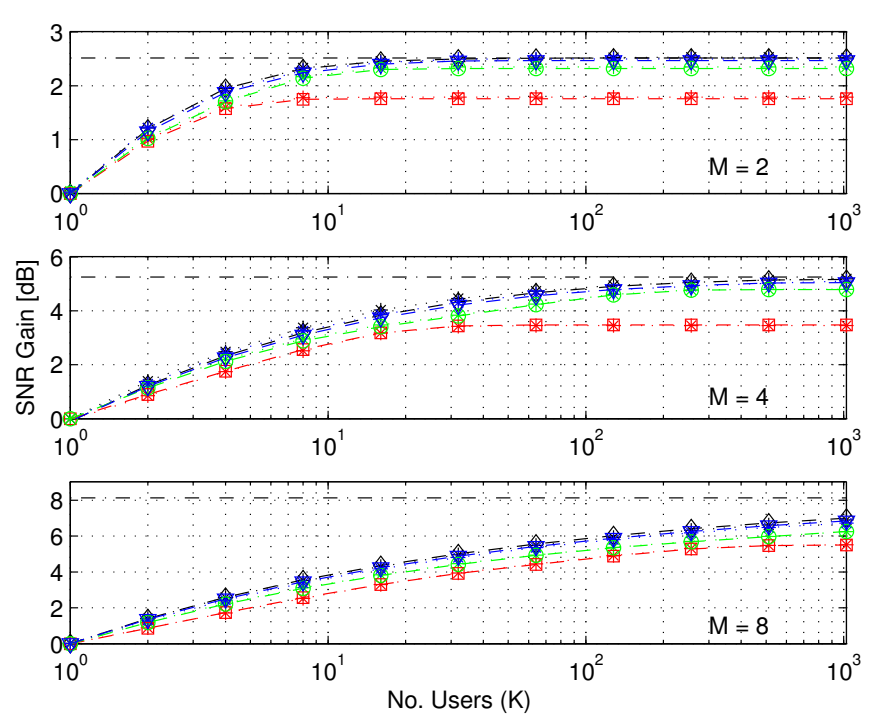

Fig. 5. SNR Gain for OCP-Proj in presence of Rayleigh fading and various numbers of BS antennas (i.e., $M=2,4,8$ ). Dashed lines with squares (' $\square$ '): $N=1$ bit. Dashed lines with circles ('o'): $N=2$ bits. Dashed lines with triangles (' $\nabla$ '): $N=3$ bits. Dashed lines with diamonds (' $\diamond$ '): $N \rightarrow \infty$ bits. Dashed-dotted lines: asymptotic upper bound. Point values ('*') were simulated to verify theoretical analysis.

of the SNR gain of (deterministic) TBF scheme increases significantly as $M$ grows. Therefore, in case of an M2M communication scenario with a moderate numbers of devices, only a small fraction of the available MUD gain is expected to be exploited, particularly when deploying large antenna arrays at the $\mathrm{BS}$ (i.e., when $M>4$ ). To overcome this limitation, multi-beam techniques can be used to increment the chance of having a device in beamforming configuration, enhancing system performance in such circumstances [23]. To implement this extension, devices would have to first estimate the projection (73) for each beam candidate, and then report to the BS the largest projection value (along with its corresponding beam index). Nevertheless, in this paper we focus our attention on the impact that feedback quantization has on a single-beam OCP systems.

\section{Achievable data rate for OCP-Proj with 1-bit feedback}

The SNR gain that OCP-Proj provides grows with both $M$ and $N$. However, this performance enhancement is obtained at the expense of incrementing the number of elements in the quantization set $\mathcal{K}$, and consequently the feedback overhead (see Section IV-B). When the number of devices $K$ grows, the probability of scheduling a device that reports a feedback codeword $\mathbf{b}_{\kappa}$ with $\kappa$ close to 1 increases. Therefore, it would be convenient to implement an adaptive strategy which dynamically controls the number of partition subsets that should be uniquely identified for given system parameters.

Let $\left\{Q_{l}: l=0, \ldots, L-1\right\}$ be the quantization regions of the performance metric, with

$$
Q_{l}=\left[q_{l}, q_{l+1}\right) \quad l=0, \ldots, L-1 ; \quad q_{0}=0 ; \quad q_{L}=1,
$$

where $\left\{q_{l}: 0, \ldots, L\right\}$ are the quantization thresholds that limit each quantization region. In practice, it is desirable to optimize these quantization levels according to

$$
\left\{\widehat{q}_{1}, \ldots, \widehat{q}_{L-1}\right\}=\arg \max _{\left\{q_{1}, \ldots, q_{L-1}\right\}} \mathbb{E}\left\{\widetilde{g}_{k^{\star}} \mid q_{1}, \ldots, q_{L-1}\right\} .
$$

In this situation, $\left\lceil\log _{2}(L)\right\rceil$ bits are necessary to uniquely identify the quantization region to which the projection metric of a given M2M device can belong. However, since feedback links are rate limited in practice, we focus our attention on using only one feedback bit per device. This feedback bit can be seen as a connection request indicator, which flags the convenience that a M2M device communicates with the BS on a given scheduling interval. We will show that the use of only one threshold, which should be appropriately adapted according to $K$, is sufficient to capture most of the MUD gain that OCP-Proj provides with ideal phase information.

Let $\mathcal{A}$ denote the event that an arbitrarily selected M2M device experiences performance metric $\kappa_{k}$ above a generic threshold $q_{1}$. Based on this, it is possible to see that

$$
\begin{aligned}
& \mathbb{E}\left\{\widetilde{g}_{k^{\star}} \mid q_{1}\right\}=\mathbb{E}\left\{\widetilde{g}_{k} \mid \mathcal{A}\right\}\left\{1-\left[F_{\kappa_{k}}\left(q_{1}\right)\right]^{K}\right\} \\
& \quad+\left\{\mathbb{E}\left\{\widetilde{g}_{k}\right\}-\mathbb{E}\left\{\widetilde{g}_{k} \mid \mathcal{A}\right\}\left[1-F_{\kappa_{k}}\left(q_{1}\right)\right]\right\}\left[F_{\kappa_{k}}\left(q_{1}\right)\right]^{K-1} \\
& \quad=\mathbb{E}\left\{\widetilde{g}_{k} \mid \mathcal{A}\right\}\left\{1-\left[F_{\kappa_{k}}\left(q_{1}\right)\right]^{K-1}\right\}+\left[F_{\kappa_{k}}\left(q_{1}\right)\right]^{K-1},(95)
\end{aligned}
$$

where

$$
\begin{gathered}
\mathbb{E}\left\{\widetilde{g}_{k} \mid \mathcal{A}\right\}=\left[\frac{1}{1-F_{\kappa_{k}}\left(q_{1}\right)}\right] \int_{q_{1}}^{1} \mathbb{E}\left\{\widetilde{g}_{k} \mid \kappa\right\} f_{\kappa_{k}}(\kappa) d \kappa, \\
\mathbb{E}\left\{\widetilde{g}_{k} \mid \kappa\right\}=\left\{\begin{array}{cc}
1+(\pi / 4)(M \kappa-1) C_{N} & \text { if } \kappa \in \mathcal{K}, \\
\times\left[2 / M+(M-2) C_{N} / M\right] & \text { otherwise. }
\end{array}\right.
\end{gathered}
$$

Then, combining (94) with (95)-(97), the final expression to obtain the optimal threshold is obtained

$$
\widehat{q}_{1}=\arg \max _{q_{1} \in \mathcal{K}} \mathbb{E}\left\{\widetilde{g}_{k^{\star}} \mid q_{1}\right\} .
$$

Similarly, we have that the conditional second moment is

$$
\begin{aligned}
& \mathbb{E}\left\{\widetilde{g}_{k^{\star}}^{2} \mid \widehat{q}_{1}\right\}=\mathbb{E}\left\{\widetilde{g}_{k}^{2} \mid \mathcal{A}\right\}\left\{1-\left[F_{\kappa_{k}}\left(\widehat{q}_{1}\right)\right]^{K}\right\} \\
& \quad+\left\{\mathbb{E}\left\{\widetilde{g}_{k}^{2}\right\}-\mathbb{E}\left\{\widetilde{g}_{k}^{2} \mid \mathcal{A}\right\}\left[1-F_{\kappa_{k}}\left(\widehat{q}_{1}\right)\right]\right\}\left[F_{\kappa_{k}}\left(\widehat{q}_{1}\right)\right]^{K-1} \\
& \quad=\mathbb{E}\left\{\widetilde{g}_{k}^{2} \mid \mathcal{A}\right\}\left\{1-\left[F_{\kappa_{k}}\left(\widehat{q}_{1}\right)\right]^{K-1}\right\}+2\left[F_{\kappa_{k}}\left(\widehat{q}_{1}\right)\right]^{K-1},(99)
\end{aligned}
$$

where

$$
\mathbb{E}\left\{\widetilde{g}_{k}^{2} \mid \mathcal{A}\right\}=\left[\frac{1}{1-F_{\kappa_{k}}\left(\widehat{q}_{1}\right)}\right] \int_{\widehat{q}_{1}}^{1} \mathbb{E}\left\{\widetilde{g}_{k}^{2} \mid \kappa\right\} f_{\kappa_{k}}(\kappa) d \kappa
$$

with

$$
\mathbb{E}\left\{\widetilde{g}_{k}^{2} \mid \kappa\right\}= \begin{cases}2-\left(2 / M^{2}\right)(M-1) C_{N-1} & \\ \times\left[1+(M / 2-1) C_{N-1}\right] & \\ +\left(8 / M^{2}\right) C_{N}(M \kappa-1) & \text { if } \kappa \in \mathcal{K}, \\ & +(\pi / 4)(M / 8)\left[1+(M / 2-1) C_{N}\right] \\ & \left.\left.+\left(1-C_{N-1}\right)\left(1+(M / 2-3 / 2) C_{N}\right)\right]\right\} \\ & +D_{1}\left(M^{2} \kappa^{2}-2 M \kappa+1\right) \\ & \end{cases}
$$




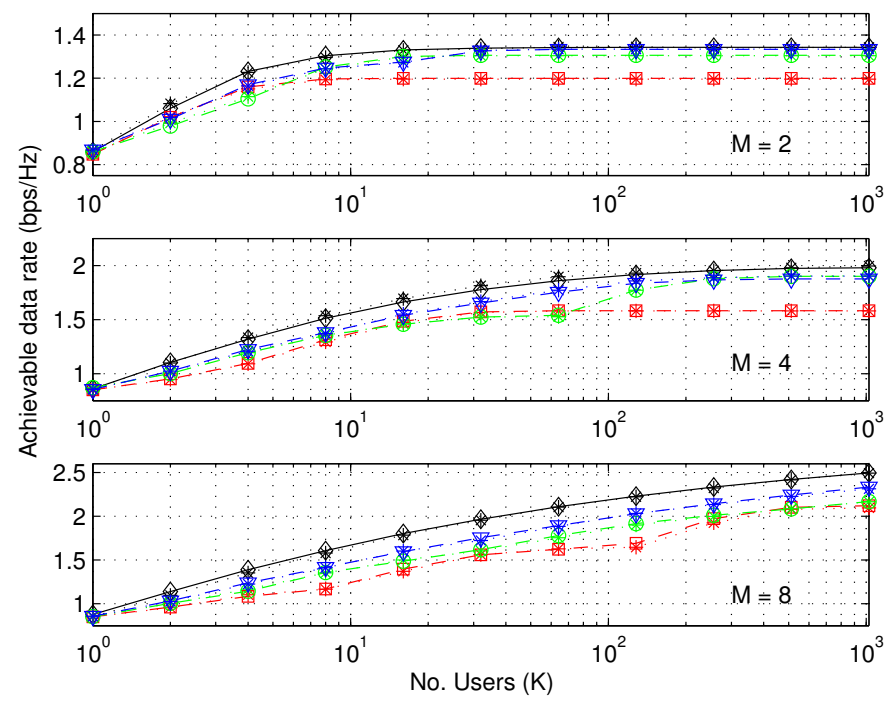

Fig. 6. Achievable data rate for OCP-Proj with 1-bit feedback in case of Rayleigh fading (i.e., $\bar{\gamma}=0 \mathrm{~dB}$ ) and various numbers of BS antennas (i.e., $M=2,4,8$ ). Dashed lines with squares (' $\square$ '): $N=1$ bit. Dashed lines with circles ('o'): $N=2$ bits. Dashed lines with triangles (' $\nabla$ '): $N=3$ bits. Dashed lines with diamonds (' $\diamond$ '): $N \rightarrow \infty$ bits. In all cases, point values ('*') were simulated to verify theoretical analysis.

and

$$
\begin{aligned}
D_{1} & =\left\{M C_{N-1}\left[1+(M / 2-1) C_{N-1}\right]\right. \\
& +M(M-2)(\pi / 4) C_{N} \\
& \times\left[C_{N-1}\left(1+(M / 2-3 / 2) C_{N}\right)+\left(C_{N} / 2\right)\right] \\
& +(M-2)(M-3)(\pi / 4)^{2} C_{N}^{3} \\
& \left.\times\left[C_{N}(M / 2-2)+2\right]\right\}\left[M\left(M^{2}-3 M+3\right)\right]^{-1} .
\end{aligned}
$$

Figure 6 presents the achievable data rate for OCP-Proj scheme as a function of the number of active devices for 1-bit signaling, when $\bar{\gamma}=0 \mathrm{~dB}$ and both $M$ and $N$ take different values. In this figure, analytical approximations for the achievable data rate were derived modeling the stochastic behavior of $\widetilde{g}_{k^{\star}}$ with a first-order corrected version of a $\chi^{2}$ distribution, whose fitting parameters were obtained with the aid of raw moment statistics presented in (95) and (99). Optimal quantization threshold $\widehat{q}_{1}$ in these curves was appropriately selected based on the number of active M2M devices in the system, see (98). Note that the effect of the threshold adaptation procedure can be observed in different parts of these curves, where the slope of the achievable data rate function changes whenever the optimal quantization threshold is updated. According to these plots, it is possible to observe that the use of OCP-Proj with 1-bit signaling allows to reap a large fraction of the MUD gain that is obtained when feedback information is reported without quantization. For example, performance loss for $N=3$ was in the order of $0.05,0.1$ and $0.2 \mathrm{bps} / \mathrm{Hz}$ when $M=2,4$ and 8 , respectively. Even though the performance degradation increases slightly for $N=2$, the use of a smaller phase quantization set $\mathcal{V}_{p}$ reduces the number of times that the threshold level $\widehat{q}_{1}$ needs to be adjusted during communication. Note that this alternative becomes advantageous in $\mathrm{M} 2 \mathrm{M}$ communication scenarios with large dynamics in the instantaneous number of active devices. In all cases, feedback overhead can be further reduced if those M2M devices that do not surpass the threshold $\widehat{q}_{1}$ remain silent [25]. Finally, when no M2M device requests communication in a given scheduling interval, there are two ways to proceed: (a) The BS can select a device at random if the goal is the maximization of the system data rate, or (b) The BS can remain silent if the goal is to keep co-channel interference and/or energy consumption low. We note that option (a) was used to obtain the simulation results in this section.

\section{CONCLUSions}

We studied the performance of a new codebook-based RBF scheme that implements opportunistic scheduling to provide a balance between mean data rate maximization and a fair share of resources among static M2M devices. In order to fulfill the requirements for M2M communication services, which include the provision of connectivity for a massive number of low-cost devices that demand extended coverage with low-power consumption, the system was designed to use a narrowband channel with multiple antennas deployed only at the BS. Moreover, slowly-varying wireless channel gains were estimated using a common high-power DL-P signal that was time-multiplexed in the BS antenna domain. The projection of the channel gain vector of the M2M device into the direction of the RBF vector of the BS was used as feedback information to be reported opportunistically.

When feedback information was reported without quantization, closed form expressions were derived to approximate the achievable data rate of three RBF schemes: OBF, OCP, and OAS. After studying the advantages and disadvantages of each alternative, we came to the conclusion that OCP provides the best trade-off between performance gain and implementation cost. So, the impact of feedback quantization was then studied when OCP scheme is combined with max-Proj scheduling.

The received SNR of the scheduled M2M device was modeled using a first-order corrected version of a $\chi^{2}$ distribution, with fitting parameters obtained from the first two raw moments of the received SNR. The derived data rate formulas were validated using numerical simulations. Our analysis revealed that the use of codebook-based OCP-Proj with 1-bit feedback allows to reap a large fraction of the MUD gain that is available in the multiuser system. It is important to highlight that this result was achieved fulfilling the requirements that have been identified to support delay-tolerant M2M services, using the infrastructure that contemporary mobile cellular networks already provide.

\section{APPENDIX I: ERROR CORRECTION FOR CHI-SQUARED DISTRIBUTION APPROXIMATION}

When approximating a generic $\operatorname{PDF} f(\gamma)$ (with unknown closed form formula) by a $\chi^{2}$ distribution with $r$ degrees of freedom and mean $\eta$, the error

$$
\varepsilon(\gamma)=f(\gamma)-\frac{1}{\Gamma\left(\frac{r}{2}\right)}\left(\frac{r}{2 \eta}\right)^{\frac{r}{2}} \gamma^{\frac{r}{2}-1} e^{-\frac{r}{2 \eta} \gamma}
$$


results. We shall express this error in terms of the raw moments $\mathbb{E}\left\{\gamma^{n}\right\}$ and the generalized Laguerre polynomials

$$
L_{k}^{(\alpha)}(u)=\frac{u^{-\alpha} e^{u}}{k !} \frac{\partial^{k}}{\partial u^{k}}\left(e^{-u} u^{k+\alpha}\right)=\sum_{i=0}^{k}\left(\begin{array}{c}
k+\alpha \\
k-i
\end{array}\right) \frac{(-u)^{i}}{i !} .
$$

These polynomials are orthogonal for real values with respect to weighting function $u^{\alpha} e^{-u}$; therefore,

$$
\int_{0}^{\infty} u^{\alpha} e^{-u} L_{k}^{(\alpha)}(u) L_{l}^{(\alpha)}(u) d u=\frac{(k+\alpha) !}{k !} \delta_{k l},
$$

where $\delta_{k l}$ is the Kronecker delta function. Due to the orthogonality property stated above, if $\gamma$ is $\chi^{2}$ distributed with $r$ degrees of freedom and mean $\eta$, then

$$
\mathbb{E}\left\{L_{k}^{(\alpha)}(\beta \gamma) L_{l}^{(\alpha)}(\beta \gamma)\right\}= \begin{cases}\frac{(k+\alpha) !}{\alpha ! k !} & k=l \\ 0 & k \neq l\end{cases}
$$

with $\alpha=\frac{r}{2}-1$ and $\beta=\frac{r}{2 \eta}$. Hence, the error can be written as a series:

$$
\varepsilon(\gamma)=\frac{\beta}{\Gamma(\alpha+1)}(\beta \gamma)^{\alpha} e^{-\beta \gamma}\left[\sum_{k=2}^{+\infty} C_{k}^{(\alpha)} L_{k}^{(\alpha)}(\beta \gamma)\right] .
$$

The series starts with $k=2$, because moments of $\varepsilon(\gamma)$ of order up to 1 are null. Now, we show how coefficients $C_{k}^{(\alpha)}$ can be expressed in terms of the (known) raw moments of $\gamma$.

Let us focus on the first-order error corrected version for $f(\gamma)$, where the approximation is obtained retaining the first nonzero term of the sum in (107), i.e.,

$$
f(\gamma) \approx \frac{\beta}{\Gamma(\alpha+1)}(\beta \gamma)^{\alpha} e^{-\beta \gamma}\left[1+C_{2}^{(\alpha)} L_{2}^{(\alpha)}(\beta \gamma)\right],
$$

where it follows from (104) that

$$
L_{2}^{(\alpha)}(\beta \gamma)=\frac{1}{2}\left[(\beta \gamma)^{2}-2(\alpha+2)(\beta \gamma)+(\alpha+1)(\alpha+2)\right] .
$$

Therefore, we only need to determine $C_{2}^{(\alpha)}$. In order to do so, it is possible to see that

$$
\begin{aligned}
\int_{0}^{\infty} L_{2}^{(\alpha)}(\beta \gamma) \varepsilon(\gamma) d \gamma= \\
\quad=\int_{0}^{\infty} L_{2}^{(\alpha)}(\beta \gamma) \frac{\beta}{\Gamma(\alpha+1)}(\beta \gamma)^{\alpha} e^{-\beta \gamma} \sum_{k=2}^{\infty}\left[C_{k}^{(\alpha)} L_{k}^{(\alpha)}(\beta \gamma)\right] d \gamma \\
\quad=C_{2}^{(\alpha)} \int_{0}^{\infty} L_{2}^{(\alpha)}(\beta \gamma) L_{2}^{(\alpha)}(\beta \gamma) \frac{\beta}{\Gamma(\alpha+1)}(\beta \gamma)^{\alpha} e^{-\beta \gamma} d \gamma \\
\quad+\sum_{k=3}^{\infty} C_{k}^{(\alpha)} \int_{0}^{\infty} L_{2}^{(\alpha)}(\beta \gamma) L_{k}^{(\alpha)}(\beta \gamma) \frac{\beta}{\Gamma(\alpha+1)}(\beta \gamma)^{\alpha} e^{-\beta \gamma} d \gamma
\end{aligned}
$$

Combining previous expression with orthogonality property introduced in (106), we have that

$$
\begin{aligned}
\int_{0}^{\infty} L_{2}^{(\alpha)}(\beta \gamma) \varepsilon(\gamma) d \gamma & =C_{2}^{(\alpha)} \frac{(\alpha+2) !}{\alpha ! 2 !} \\
& =\frac{1}{2} C_{2}^{(\alpha)}(\alpha+2)(\alpha+1) .
\end{aligned}
$$

Following an alternative analysis, i.e., replacing $L_{2}^{(\alpha)}(\beta \gamma)$ by expression (109),

$$
\begin{aligned}
\int_{0}^{\infty} L_{2}^{(\alpha)}(\beta \gamma) \varepsilon(\gamma) d \gamma & =\frac{1}{2} \int_{0}^{\infty}(\beta \gamma)^{2} \varepsilon(\gamma) d \gamma \\
& -(\alpha+2) \int_{0}^{\infty}(\beta \gamma) \varepsilon(\gamma) d \gamma \\
& +\frac{1}{2}(\alpha+2)(\alpha+1) \int_{0}^{\infty} \varepsilon(\gamma) d \gamma
\end{aligned}
$$

The last two integrals vanish, because the moments of $\varepsilon(\gamma)$ of order up to 1 are null. Therefore,

$$
\begin{aligned}
\int_{0}^{\infty} L_{2}^{(\alpha)}(\beta \gamma) \varepsilon(\gamma) d \gamma & =\frac{1}{2}\left[\int_{0}^{\infty}(\beta \gamma)^{2} f(\gamma) d \gamma\right. \\
& \left.-\int_{0}^{\infty}(\beta \gamma)^{2} \frac{\beta}{\Gamma(\alpha+1)}(\beta \gamma)^{\alpha} e^{-\beta \gamma} d \gamma\right] \\
& =\frac{\beta^{2}}{2}\left[\int_{0}^{\infty} \gamma^{2} f(\gamma) d \gamma\right. \\
& \left.-\int_{0}^{\infty} \gamma^{2} \frac{\beta}{\Gamma(\alpha+1)}(\beta \gamma)^{\alpha} e^{-\beta \gamma} d \gamma\right] \\
& =\frac{\beta^{2}}{2}\left[\mathbb{E}\left\{\gamma^{2}\right\}-\frac{(\alpha+1)(\alpha+2)}{\beta^{2}}\right] .
\end{aligned}
$$

Combining (111) with (113),

$$
C_{2}^{(\alpha)}=\frac{\beta^{2} \mathbb{E}\left\{\gamma^{2}\right\}}{(\alpha+1)(\alpha+2)}-1
$$

results. Replacing the latter expression in (108), final first-order corrected expression is obtained:

$$
\begin{aligned}
f(\gamma) & \approx \frac{\beta}{\Gamma(\alpha+1)}(\beta \gamma)^{\alpha} e^{-\beta y}\left(a_{2} \gamma^{2}+a_{1} \gamma+a_{0}\right) \\
a_{2} & =\left[\frac{\beta^{2}}{2}\right]\left[\frac{\beta^{2} \mathbb{E}\left\{\gamma^{2}\right\}}{(\alpha+1)(\alpha+2)}-1\right] \\
a_{1} & =-2 \frac{(\alpha+2)}{\beta} a_{2}, \quad a_{0}=-\frac{1}{2} \frac{(\alpha+1)}{\beta} a_{1}+1 .
\end{aligned}
$$

\section{ACKNOWLEDGEMENTS}

This material is partly based upon works supported by the Academy of Finland under Grants 284634 and 284811, the Spanish Government under Project TEC2014-59255-C3-1-R, and the Catalan Government under Grant 2014SGR1567.

\section{REFERENCES}

[1] T. Taleb and A. Kunz, "Machine type communications in 3GPP networks: potential, challenges, and solutions," IEEE Commun. Mag. vol. 50, no. 3, pp. 178-184, Mar. 2012.

[2] D. Astely, E. Dahlman, G. Fodor, S. Parkvall, and J. Sachs, "LTE release 12 and beyond," IEEE Commun. Mag., vol. 51, no. 7, pp. 154 160, Jul. 2013.

[3] M. Hasan, E. Hossain, and D. Niyato, "Random access for machineto-machine communication in LTE-advanced networks: issues and approaches," IEEE Commun. Mag., vol. 51, no. 6, pp. 86-93, Jun. 2013.

[4] P. Viswanath, D. N. C. Tse, and R. Laroia, "Opportunistic beamforming using dumb antennas," IEEE Trans. Inf. Theory, vol. 48, no. 6, pp. 12771294, Jun. 2002.

[5] J. Zhu and H.-C. Yang, "Performance analysis of low-complexity dualcell random beamforming transmission with user scheduling," EURASIP J. Wireless Communications and Networking, vol. 2011, pp. 1-11, Dec. 2011. 
[6] F. Floren, O. Edfors, and B.-A. Molin, "The effect of feedback quantization on the throughput of a multiuser diversity scheme," in Proc. IEEE Global Telecommun. Conf., vol. 1, Dec. 2003, pp. 497-501.

[7] S. Sanayei and A. Nosratinia, "Exploiting multiuser diversity with only 1-bit feedback," in Proc. IEEE Wireless Commun. and Netw. Conf., vol. 2, Mar. 2005, pp. 978-983.

[8] O. Özdemir and M. Torlak, "Optimum feedback quantization in an opportunistic beamforming scheme," IEEE Trans. Wireless Commun. vol. 9, no. 5, pp. 1584-1593, May 2010 .

[9] M. Xia, W. Wen, and S.-C. Kim, "Opportunistic cophasing transmission in MISO systems," IEEE Trans. Commun., vol. 57, no. 12, pp. 3764 3770, Dec. 2009.

[10] N. Sharma and L. H. Ozarow, "A study of opportunism for multipleantenna systems," IEEE Trans. Inf. Theory, vol. 51, no. 5, pp. 1804 1814, May 2005.

[11] A. A. Dowhuszko, G. Corral-Briones, J. Hämäläinen, and R. Wichman, "On the analysis and design of practical quantization for opportunistic beamforming," in Proc. IEEE Int. Conf. on Commun., May 2008, pp. 5133-5139.

[12] M. Sharif and B. Hassibi, "On the capacity of MIMO broadcast channels with partial side information," IEEE Trans. Inf. Theory, vol. 51, no. 2, pp. 506-522, Feb. 2005.

[13] H.-C. Yang, P. Lu, H.-K. Sung, and Y.-C. Ko, "Exact sum-rate analysis of MIMO broadcast channels with random unitary beamforming," IEEE Trans. Commun., vol. 59, no. 11, pp. 2982-2986, Nov. 2011.

[14] Y. Huang and B. D. Rao, "Closed form sum rate of random beamforming," IEEE Commun. Letters, vol. 16, no. 5, pp. 630-633, May 2012.

[15] M. Xia, Y.-C. Wu, and S. Aissa, "Non-orthogonal opportunistic beamforming: Performance analysis and implementation," IEEE Trans. Wireless Commun, vol. 11, no. 4, pp. 1424-1433, Apr. 2012.

[16] A. A. Dowhuszko, G. Corral-Briones, J. Hämäläinen, and R. Wichman, "On throughput-fairness tradeoff in virtual MIMO systems with limited feedback," EURASIP J. Wireless Communications and Networking, vol. 2009, pp. 1-17, Jan. 2009.

[17] M. Abramowitz and I. A. Stegun, Handbook of mathematical functions: with formulas, graphs, and mathematical tables. Dover Publications, 1970.

[18] C. K. Au-Yeung and D. J. Love, "On the performance of random vector quantization limited feedback beamforming in a MISO system," IEEE Trans. Wireless Commun., vol. 6, no. 2, pp. 458-462, Feb. 2007.

[19] R. Garrappa, "Some formulas for sums of binomial coefficients and gamma functions," International Mathematical Forum, vol. 2, no. 15, pp. 725-733, 2007.

[20] I. S. Gradshteyn and I. M. Ryzhik, Table of Integrals, Series and Products, 5th ed. Academic Press Inc, 1994.

[21] K. Pearson, "The problem of the random walk," Nature, vol. 72, p. 294, Jul. 1905.

[22] B. B. Hughes, Random Walks and Random Environments, Vol. 1: Random Walks. Oxford University Press, Mar. 1995.

[23] J. Kang, I.-K. Choi, D.-S. Kwon, and C. Lee, "An opportunistic beamforming technique using a quantized codebook," in Proc. IEEE Veh. Tech. Conf. Spring, Apr. 2007, pp. 1647-1651.

[24] O. Özdemir and M. Torlak, "Performance of opportunistic beamforming with quantized feedback," in Proc. IEEE Global Telecommun. Conf., Nov. 2006, pp. 1-5.

[25] T. Samarasinghe, H. Inaltekin, and J. S. Evans, "Optimal selective feedback policies for opportunistic beamforming," IEEE Trans. Inform. Theory, vol. 59, no. 5, pp. 2897-2913, May 2013.

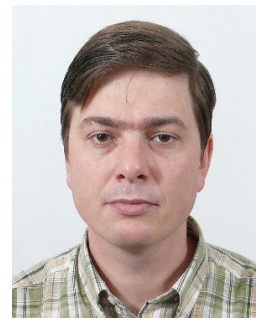

Alexis A. Dowhuszko received the telecommunications engineer degree from Blas Pascal University, Cordoba, Argentina, in 2002, and the Ph.D. degree in engineering sciences from the National University of Cordoba, Argentina, in 2010. From 2010 to 2015, he was a Post-Doctoral Researcher at the Department of Communications and Networking, Aalto University, Finland. He joined the Catalan Telecommunications Technology Center (CTTC) in early 2016 to carry out research on disruptive communication concepts, technologies, and architectures in the context of $5 \mathrm{G}$ networks and beyond. Dowhuszko has authored or co-authored 15 journa papers, 25 conference articles, one book chapter and one patent application.

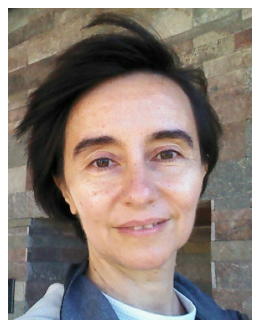

Graciela Corral-Briones received the Ph.D. degree from Universidad Nacional de Córdoba (UNC), Argentina, in 2007. From 2006 to 2008, she worked at ClariPhy Argentina S.A. as senior research engineer She is currently an Associate Professor at UNC and a senior researcher at the National Research Council (CONICET) of Argentina. She has been involved in several national and international research projects in the fields of digital communications. Her research interests include signal processing and performance analysis for wireless communication systems.

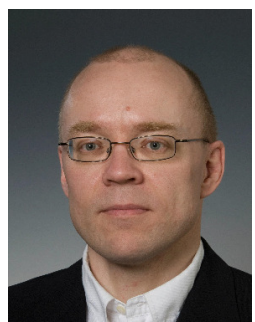

Jyri Hämäläinen received his M.Sc. and $\mathrm{Ph} . \mathrm{D}$. degrees from University of Oulu, Finland, in 1992 and 1998, respectively. From 1999 to the end of 2007 he was with Nokia where he worked on various aspects of mobile communication systems. Since 2008 he has been a Professor in Aalto University, School of Electrical Engineering, where he is currently serving as a Dean. His research interests include small cells, multi-antenna transmission and reception techniques, scheduling, relays, and design and analysis of future wireless networks in genera Hämäläinen is author or co-author of over 180 scientific publications and 36 US patents or patent applications.

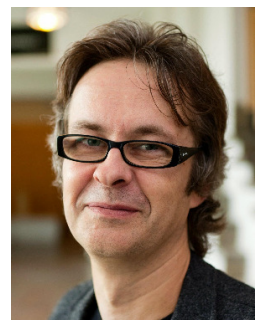

Risto Wichman received his M.Sc. and D.Sc. (Tech) degrees in digital signal processing from Tampere University of Technology, Tampere, Finland, in 1990 and 1995, respectively. From 1995 to 2001, he worked at Nokia Research Center as a senior research engineer. In 2002, he joined the Department of Signal Processing and Acoustics, Aalto University School of Electrical Engineering, Finland, where he is a full professor since 2008 . His research interests include signal processing techniques for wireless communication systems 